\title{
Ray Scattering by an Arbitrarily Oriented Spheroid .I. Diffraction and Specular Reflection
}

James A. Lock

Cleveland State University, j.lock@csuohio.edu

Follow this and additional works at: https://engagedscholarship.csuohio.edu/sciphysics_facpub

Part of the Physics Commons

How does access to this work benefit you? Let us know!

\section{Publisher's Statement}

This paper was published in Applied Optics and is made available as an electronic reprint with the permission of OSA. The paper can be found at the following URL on the OSA website: http://www.opticsinfobase.org/ao/abstract.cfm?URI=ao-35-3-500. Systematic or multiple reproduction or distribution to multiple locations via electronic or other means is prohibited and is subject to penalties under law.

\section{Original Citation}

Lock, James A. "Ray Scattering by an Arbitrarily Oriented Spheroid .I. Diffraction and Specular Reflection." Applied Optics 35 (1996): 500-514.

\section{Repository Citation}

Lock, James A., "Ray Scattering by an Arbitrarily Oriented Spheroid .I. Diffraction and Specular Reflection" (1996). Physics Faculty Publications. 41.

https://engagedscholarship.csuohio.edu/sciphysics_facpub/41

This Article is brought to you for free and open access by the Physics Department at EngagedScholarship@CSU. It has been accepted for inclusion in Physics Faculty Publications by an authorized administrator of EngagedScholarship@CSU. For more information, please contact library.es@csuohio.edu. 


\title{
Ray scattering by an arbitrarily oriented spheroid. I. Diffraction and specular reflection
}

\author{
James A. Lock
}

\begin{abstract}
Diffraction and reflection of an arbitrarily polarized plane wave by an arbitrarily oriented spheroid in the short-wavelength limit are considered in the context of ray theory. A closed-form solution for both diffraction and reflection is obtained, and the polarization character of the diffracted plus reflected electric field is obtained. It is found that the magnitude of the reflected electric field is multivalued for forward scattering. This is interpreted in terms of the variation of the spheroid's Gaussian curvature at the points where grazing ray incidence occurs.
\end{abstract}

\section{Introduction}

The mathematical solution of the electromagnetic boundary-value problem of a plane wave scattered by a dielectric sphere has long been known, ${ }^{1-3}$ and stable computer programs for calculating the farzone scattered intensity are widely available., ${ }^{4,5}$ Much progress has been made on the related electromagnetic boundary-value problem of a plane wave scattered by an arbitrarily oriented dielectric spheroid. The plane-wave-spheroid problem has been solved exactly with the method of separation of variables by the use of radial and angular spheroidal wave functions, ${ }^{6-8}$ with the extended boundarycondition method otherwise known as the T-matrix method, ,, 10 and with the boundary-condition-matching method. ${ }^{11}$ A computer program for calculating spheroid scattering is also widely available. ${ }^{10}$ But certain computational problems still remain.

In each of the three exact methods for solving the plane-wave-spheroid problem, the incident, scattered, and interior waves are expanded in an infinite series of partial waves. Imposition of the boundary conditions at the spheroid surface generates an infinite system of linear equations that must be truncated and then solved by the use of matrix inversion. The unknowns in this system of equations are the partial-wave interior amplitudes and

The author is with the Department of Physics, Cleveland State University, Cleveland, Ohio 44115.

Received 30 January 1995; revised manuscript received 1 June 1995.

0003-6935/96/0500-15\$06.00/0

(c) 1996 Optical Society of America scattering amplitudes. In the method of Refs. 6-8 the elements of the coefficient matrix are integrals of functions derived from the angular spheroidal wave functions and must be evaluated numerically. In the methods of Refs. 9-11, the elements of the coefficient matrix are integrals of functions derived from vector spherical harmonics and must also be evaluated numerically. In each of the three methods, the inversion of the coefficient matrix becomes numerically ill conditioned for large spheroid-size parameters and large spheroid eccentricities. ${ }^{7,11,12}$ This ill conditioning effectively limits the utility of the exact solution methods to spheroid-size parameters less than approximately 35 .

For large spheroid-size parameters there are a number of approximate methods for calculating the far-zone scattered intensity. These include the physical optics model, ${ }^{13-15}$ the eikonal method otherwise known as anomalous diffraction, ${ }^{12,16-18}$ the geometric theory of diffraction, ${ }^{19}$ catastrophe optics methods, ${ }^{20}$ and ray theory. Each of these approximate methods is successful in describing certain features of the far-zone scattered intensity. In the physical optics model, Maxwell's equations are combined to obtain the electric field at any point exterior to the spheroid in terms of an integral of the fields on the spheroid surface. The surface fields in turn are evaluated approximately with flux conservation, Snell's law, and the Fresnel coefficients for reflection and transmission of light rays undergoing multiple internal reflections inside the spheroid. The physical optics model has the advantage that it is reasonably accurate over the entire range of scattering angles. But it has the disadvantage that to date, 
calculations have been limited to only two spheroid orientations and to size parameters less than 30.

The eikonal method assumes that the plane wave is transmitted through the spheroid without refraction at its surface. The spheroid produces only a position-dependent phase delay of the transmitted wave front. The transmitted electric field in the spheroid's exit plane is then Fraunhofer diffracted into the far zone. The specular-reflection surface waves are also included in an approximate way. For scattering by a sphere, ${ }^{16-18}$ the eikonal model gives accurate results for scattering angles less than $60^{\circ}$. For scattering by a spheroid, ${ }^{12}$ this model has been used primarily to obtain the scattering cross section. Its disadvantage is that the method cannot be applied to large angle scattering, and because it is based on scalar waves, it cannot provide polarization information. For scattering by a sphere this is not important because the difference between the two polarizations is small for scattering in the forward hemisphere. But spheroid scattering exhibits crosspolarization effects, which cannot be calculated with this method.

The geometrical theory of diffraction is limited to scattering by an impenetrable spheroid because it includes only reflection, diffraction, and the specularreflection surface waves. To date, it has been used only for a point source on the spheroid major axis and a plane wave traveling along the spheroid major axis. The far-zone electric field has been calculated only for backscattering.

The catastrophe optics method is a generalization of Airy theory for scattering by a sphere. ${ }^{21,22}$ For light rays making one or more internal reflections within the spheroid, it has been found that the far-zone rainbow caustics are produced by the coalescence of a large number of rays and correspond to a higher-order optical catastrophe than the rainbow for sphere scattering. ${ }^{23}$ In the catastrophe optics method, the curvature of the wave front exiting the spheroid in the vicinity of the rainbow scattering angle is obtained and then Fraunhofer diffracted into the far zone. The resulting diffraction integral describes both the optical caustic and the diffraction structure that surrounds it. This method has proven to be quite successful in describing the observed caustic structure of the rainbow for spheroid scattering. ${ }^{24,25}$ But it has not been applied to scattering angles far from the caustic.

For scattering by a sphere in the short-wavelength limit, ray theory also gives a good approximation to the far-zone intensity as long as one does not get too close to the rainbow scattering angles or the forwardscattering and backscattering glory regions. In particular, if only Fraunhofer diffraction, reflection, and transmission are taken into account, good comparisons with the results of the exact Lorenz-Mie theory for plane-wave scattering have been obtained ${ }^{26,27}$ for scattering angles as large as $50^{\circ}$ and for sphere-size parameters as small as 30. Ray theory has been applied to spheroid scattering when the plane wave is incident along the spheroid major axis. ${ }^{28,29}$ In this case the trajectory of a given light ray is confined to a single plane, thus considerably simplifying the treatment and eliminating all cross-polarization effects. Ray theory has also been applied to spheroid scattering for the purpose of examining the role of one-internal-reflection rays and two-internal-reflection rays in rainbow formation when the plane wave is incident along one of the spheroid minor axes. ${ }^{30-35}$

In this paper and in a companion paper, ${ }^{36}$ ray theory is examined for an arbitrarily polarized plane wave that is incident upon an arbitrarily oriented spheroid. The far-zone electric field is derived for diffraction, reflection, and transmission, thus limiting the applicability of our results to forwardhemisphere scattering by a transparent spheroid. Because a closed-form solution is obtained for diffraction plus reflection, the results should also be an accurate approximation for scattering by a conducting spheroid in the short-wavelength limit over the entire range of scattering angles. Unlike the eikonal model or the geometric theory of diffraction, surface-wave contributions to scattering are not considered. The Fock transition of the transmitted electric field in the vicinity of the critical angle for total internal reflection is not considered either. But because the polarization vector is tracked, the cross-polarized scattering contributions to the farzone intensity are obtained.

The body of this paper is organized as follows. In Section 2 the notation is defined and a number of geometric properties of the arbitrarily oriented spheroid are examined. In Subsection 3.A. a general expression is obtained for the magnitude of the electric field of either the reflected light rays or the light rays transmitted after $p-1$ internal reflections with $p \geq 1$. In Subsection 3.B. this result is used to obtain the magnitude of the reflected electric field in closed form. In Subsection 3.C. Fraunhofer diffraction and Babinet's principle are used to obtain the magnitude of the diffracted electric field. In Subsection 3.D., analytic geometry is used to obtain the phase of the reflected electric field, and in Subsection 3.E. the phase of the diffracted electric field is obtained. In Subsections 3.F. and 3.G. the polarization directions of the reflected and the diffracted electric fields, respectively, are obtained. In Subsection 3.H. the expressions for the reflected and the diffracted electric fields are combined. Last, in Section 4 comments on various properties of the reflected electric field are presented, and the special cases of end-on and side-on incidence are examined. The magnitude, phase, and polarization of the transmitted electric field are derived in the companion paper. $^{36}$

\section{Spheroid Geometry}

Consider a plane wave of wavelength $\lambda$ and angular frequency $\omega$ traveling along the positive $z$ axis of an $x y z$ coordinate system fixed in the laboratory reference frame. The electric field of the plane wave 
makes an angle $\chi$ with the positive $x$ axis and is given by

$$
\mathbf{E}_{\text {inc }}=E_{0}\left(\cos \chi \hat{u}_{x}+\sin \chi \hat{u}_{g}\right) \exp (i k z-i \omega t),
$$

where $E_{0}$ is the incident electric-field strength and the wave number $k$ is given by

$$
k=\frac{2 \pi}{\lambda} .
$$

In ray theory the incident plane wave is equivalent to a family of parallel light rays traveling in phase with each other. Each incident ray is characterized by the lab coordinates $x, y$. Light is scattered by the spheroid in the $\Theta, \Phi$ direction referenced with respect to the $x y z$ coordinate axes. The light-scattering geometry is illustrated in Fig. 1.

Each ray-theory contribution to the far-zone scattered electric field in the $\Theta, \Phi$ direction is of the form

$$
\begin{aligned}
\mathbf{E}_{\text {scattered }}(\Theta, \Phi)= & \frac{i E_{0}}{k R} \exp (i k R-i \omega t) S(\Theta, \Phi) \\
& \times \exp [i \delta(\Theta, \Phi)] \boldsymbol{\epsilon}(\Theta, \Phi),
\end{aligned}
$$

where $R$ is the distance from the origin to the far-zone observer, $S(\Theta, \Phi)$ is the magnitude of the electric-field contribution obtained by temporarily ignoring the Fresnel reflection and transmission coefficients of the rays as they interact with the surface of the spheroid, $\delta(\Theta, \Phi)$ is the phase of the electric-field contribution with respect to that of a suitably chosen reference ray, and $\epsilon(\Theta, \Phi)$ is the polarization vector of the electric-field contribution and contains the Fresnel reflection and transmission coefficients.

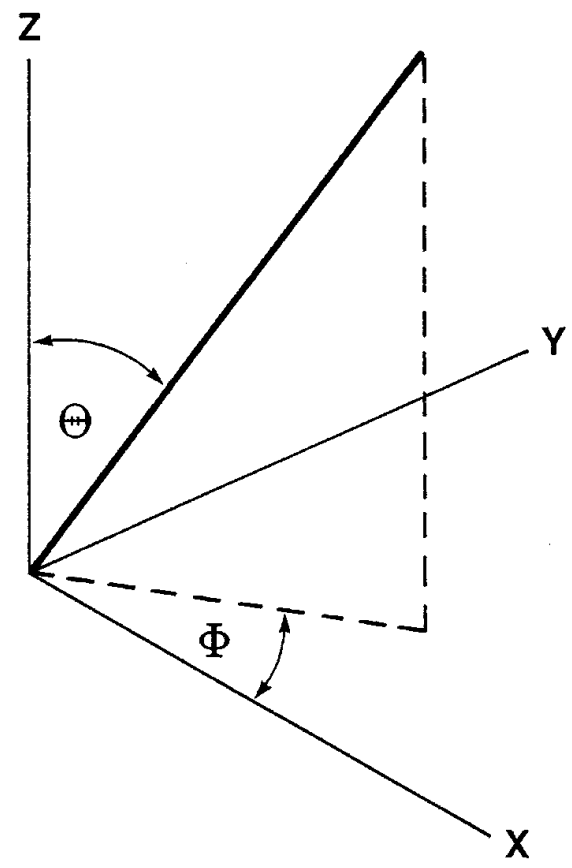

Fig. 1. Laboratory coordinate system $x y z$ and the scattering angles $\Theta, \Phi$.
Consider a dielectric spheroid of refractive index $n$ whose surface is given by

$$
\frac{x^{\prime \prime 2}}{a^{2}}+\frac{y^{\prime \prime 2}}{a^{2}}+\frac{z^{\prime 2}}{b^{2}}=1,
$$

where $x^{\prime \prime} y^{\prime \prime} z^{\prime \prime}$ are a set of coordinate axes attached to the spheroid. This is shown in Fig. 2. The spheroid is oriented so that the $z^{\prime \prime}$ axis is in the $\theta, \phi$ direction of the $x y z$ lab coordinate system. All orientations of the spheroid are within the range $0 \leq \theta \leq \pi / 2$ and $0 \leq \phi<2 \pi$. When $\theta=0$, the spheroid major axis is parallel to the incident light rays. This orientation is called end-on incidence, and as is shown below, the trajectory of a ray for this orientation is confined to a single plane. When $\theta=$ $\pi / 2$, one of the spheroid minor axes is parallel to the incident light rays. This orientation is called side-on incidence.

The equation of the spheroid surface in terms of the lab coordinates,

$$
\begin{aligned}
& z^{2}\left(\frac{\sin ^{2} \theta}{a^{2}}+\frac{\cos ^{2} \theta}{b^{2}}\right)+2 z\left(\frac{1}{b^{2}}-\frac{1}{a^{2}}\right)(x \cos \phi+y \sin \phi) \\
& \times \sin \theta \cos \theta+\frac{x^{2}}{a^{2}}\left(\cos ^{2} \theta \cos ^{2} \phi+\sin ^{2} \phi\right) \\
& +\frac{x^{2}}{b^{2}} \sin ^{2} \theta \cos ^{2} \phi+\frac{y^{2}}{a^{2}}\left(\cos ^{2} \theta \sin ^{2} \phi+\cos ^{2} \phi\right) \\
& +\frac{y^{2}}{b^{2}} \sin ^{2} \theta \sin ^{2} \phi \\
& +2 x y\left(\frac{1}{b^{2}}-\frac{1}{a^{2}}\right) \sin ^{2} \theta \sin \phi \cos \phi=1,
\end{aligned}
$$

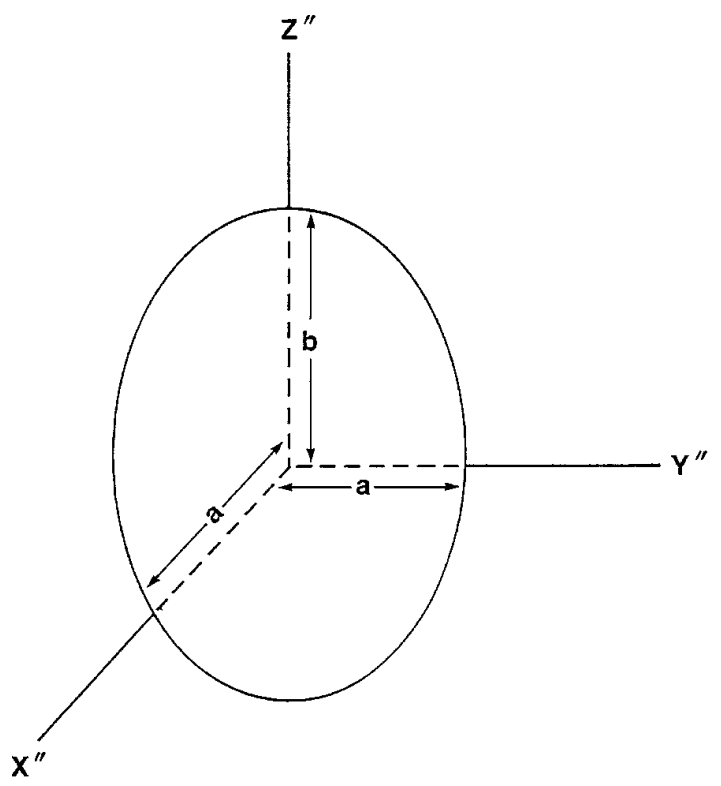

Fig. 2. Coordinate system $x^{\prime \prime} y^{\prime \prime} z^{\prime \prime}$ attached to the spheroid. The spheroid has the radius $a$ in the $x^{\prime \prime}$ and $y^{\prime \prime}$ directions and the radius $b$ in the $z^{\prime \prime}$ direction. 
is complicated. Equation (5) becomes simpler in a second coordinate system $x^{\prime} y^{\prime} z^{\prime}$ rotated with respect to the $x y z$ system by the angle $\phi$ about the $z$ axis. The two sets of lab coordinates are related by

$$
\begin{aligned}
& x^{\prime}=x \cos \phi+y \sin \phi, \\
& y^{\prime}=-x \sin \phi+y \cos \phi, \\
& z^{\prime}=z .
\end{aligned}
$$

This rotation puts the $z^{\prime \prime}$ major axis of the spheroid directly above the $x^{\prime}$ axis, as is shown in Fig. 3. In the rotated lab coordinate system, the equation of the spheroid surface is

$$
\left.\begin{array}{l}
z_{\text {upper }}{ }^{\prime} \\
z_{\text {lower }}{ }^{\prime}
\end{array}\right\}=w x^{\prime} \pm \frac{a b}{A}\left(1-\frac{x^{\prime 2}}{A^{2}}-\frac{y^{\prime 2}}{B^{2}}\right)^{1 / 2}
$$

where $z_{\text {lower }}$ is the surface of the spheroid struck by the incident light rays, i.e., the lit side, and $z_{\text {upper }}$ ' is the shadowed side, and where

$$
w=\sin \theta \cos \theta\left(\frac{b^{2}-a^{2}}{A^{2}}\right) .
$$

For end-on and side-on incidence $w=0$. The quantities $A$ and $B$ in Eq. (7) are given by

$$
\begin{aligned}
& A=\left(b^{2} \sin ^{2} \theta+a^{2} \cos ^{2} \theta\right)^{1 / 2}, \\
& B=a
\end{aligned}
$$

and have the following geometric meaning. The projection of the arbitrarily oriented spheroid onto the $x^{\prime} y^{\prime}$ plane is the ellipse

$$
\frac{x^{\prime 2}}{A^{2}}+\frac{y^{\prime 2}}{B^{2}}=1 .
$$

The quantity $A$ is the radius of the elliptical projec-

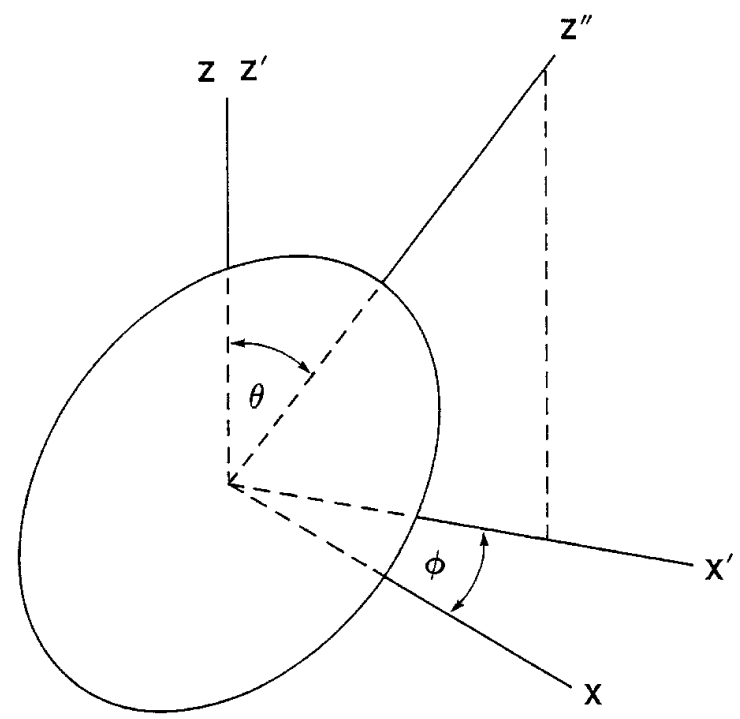

Fig. 3. Arbitrarily oriented spheroid with respect to the original and the rotated lab coordinate systems. tion along the $x^{\prime}$ axis and $B$ is the radius along the $y^{\prime}$ axis. This ellipse serves as the boundary of the effective aperture for Fraunhofer diffraction by the spheroid.

A further simplification for the shape of the spheroid surface results from converting the rotated labframe rectangular coordinates $x^{\prime}, y^{\prime}$ to the elliptical coordinates $r^{\prime}, \xi^{\prime}$ given by

$$
\begin{aligned}
& x^{\prime}=A r^{\prime} \cos \xi^{\prime}, \\
& y^{\prime}=B r^{\prime} \sin \xi^{\prime},
\end{aligned}
$$

where $0 \leq r^{\prime} \leq 1$ and $0 \leq \xi^{\prime}<2 \pi$. Equation (7) then becomes

$$
\left.\begin{array}{c}
z_{\text {upper }}{ }^{\prime} \\
z_{\text {lower }}^{\prime}
\end{array}\right\}=w A r^{\prime} \cos \xi^{\prime} \pm \frac{a b}{A}\left(1-r^{\prime 2}\right)^{1 / 2} .
$$

Instead of being parameterized by the coordinates $x$, $y$, each incident ray may be thought of as being parameterized by $r^{\prime}, \xi^{\prime}$. The rays that graze the edge of the spheroid have $r^{\prime}=1$.

Last, in Fig. 4 we show the outward normals to the upper and lower spheroid surfaces. Referenced with respect to the rotated lab coordinates $x^{\prime} y^{\prime} z^{\prime}$, they are

$$
\hat{m}^{\prime}=\frac{\left(\frac{\partial z_{\text {lower }}}{\partial x^{\prime}}\right) \hat{u}_{x}{ }^{\prime}+\left(\frac{\partial z_{\text {lower }}}{\partial y^{\prime}}\right) \hat{u}_{y}{ }^{\prime}-\hat{u}_{z}{ }^{\prime}}{\left[\left(\frac{\partial z_{\text {lower }}}{\partial x^{\prime}}\right)^{2}+\left(\frac{\partial z_{\text {lower }}}{\partial y^{\prime}}\right)^{2}+1\right]^{1 / 2}}
$$
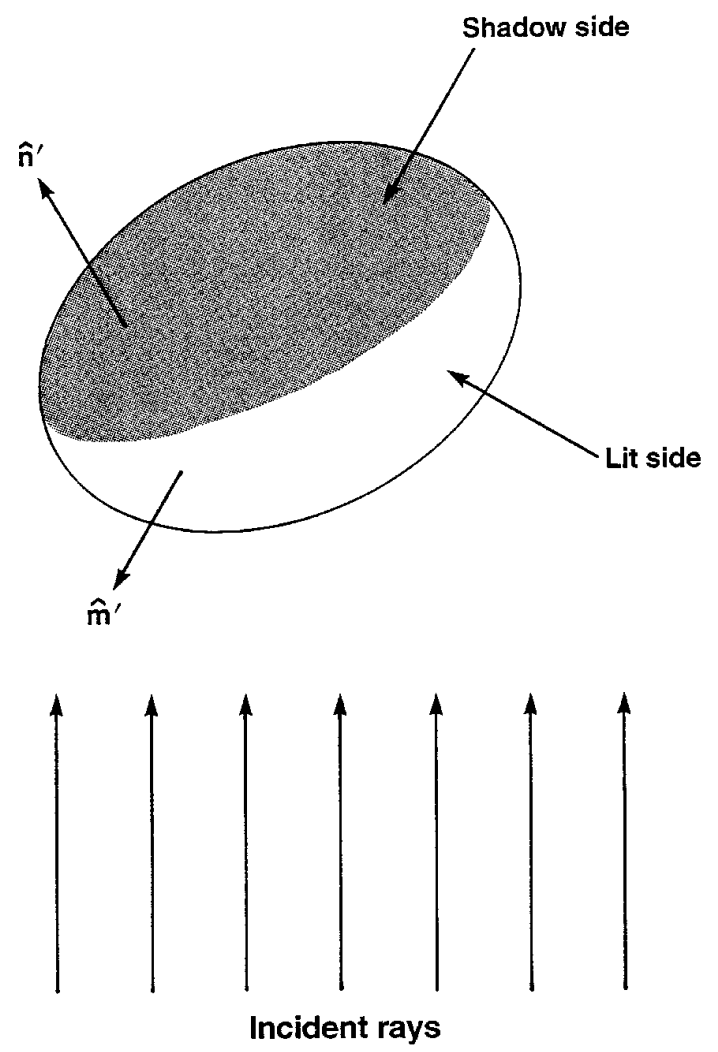

Fig. 4. Outward unit normals $\hat{m}^{\prime}$ and $\hat{n}^{\prime}$ on the lit side and the shadowed side of the spheroid, respectively. 
for the lower-surface normal and

$$
\hat{n}^{\prime}=\frac{-\left(\frac{\partial z_{\text {upper }}}{\partial x^{\prime}}\right) \hat{u}_{x}{ }^{\prime}-\left(\frac{\partial z_{\text {upper }}}{\partial y^{\prime}}\right) \hat{u}_{y}{ }^{\prime}+\hat{u}_{z}{ }^{\prime}}{\left[\left(\frac{\partial z_{\text {upper }}{ }^{\prime}}{\partial x^{\prime}}\right)^{2}+\left(\frac{\partial z_{\text {upper }}{ }^{\prime}}{\partial y^{\prime}}\right)^{2}+1\right]^{1 / 2}}
$$

for the upper-surface normal.

\section{A. Flux Conservation and the Magnitude of the Deflected Electric Field}

Consider a thin bundle of incident geometric light rays centered on the point $r^{\prime}, \xi^{\prime}$, as is shown in Fig. 5. The cross-sectional area of the incident bundle is $A B r^{\prime} \mathrm{d} r^{\prime} \mathrm{d} \xi^{\prime}$, and the power contained in the bundle is

$$
P_{\mathrm{inc}}=\frac{E_{0}^{2}}{2 \mu_{0} c} A B r^{\prime} \mathrm{d} r^{\prime} \mathrm{d} \xi^{\prime}
$$

where $\mu_{0}$ is the permeability of free space and $c$ is the speed of light. After being deflected by either specular reflection or transmission following $p-1$ internal reflections with $p \geq 1$, the incident bundle is converted into a diverging bundle of outgoing light rays centered about the scattering angles $\Theta, \Phi$, as is shown in Fig. 5. The cross-sectional area of the

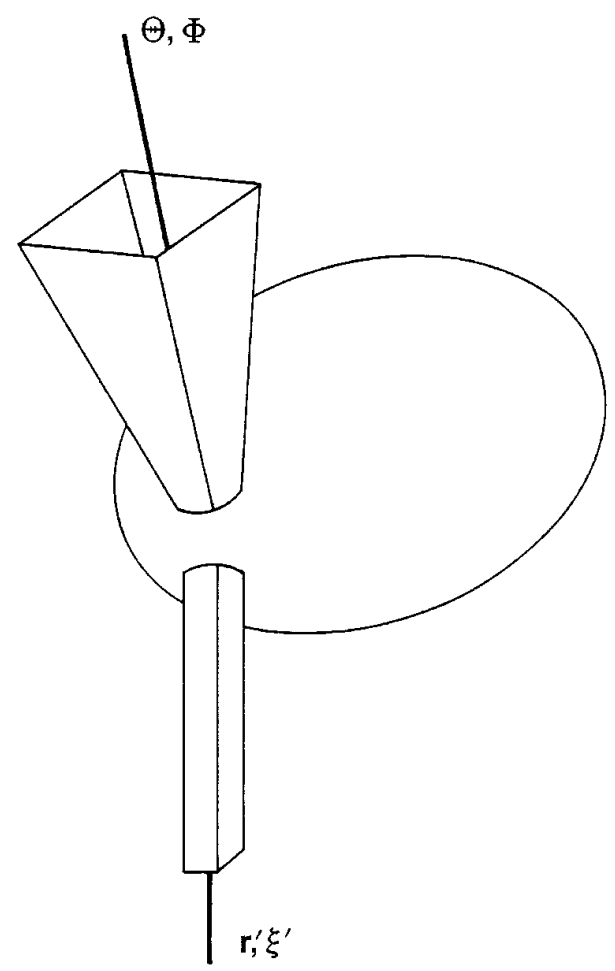

Fig. 5. Incident and outgoing flux tubes. The incident flux tube is centered about the point $r^{\prime}, \xi^{\prime}$ and has the cross-sectional area $A B r^{\prime} \mathrm{d} r^{\prime} \mathrm{d} \xi^{\prime}$. The outgoing flux tube is centered about the scattering angles $\Theta, \Phi$ and has the cross-sectional area $R^{2} \Delta \sin \Theta \mathrm{d} r^{\prime} \mathrm{d} \xi^{\prime}$, where $\Delta$ is given in Eq. (17). outgoing bundle in the far zone is $R^{2} \Delta \sin \Theta \mathrm{d} r^{\prime} \mathrm{d} \xi^{\prime}$, where $^{37}$

$$
\Delta=\left|\begin{array}{ll}
\frac{\partial \Theta}{\partial r^{\prime}} & \frac{\partial \Theta}{\partial \xi^{\prime}} \\
\frac{\partial \Phi}{\partial r^{\prime}} & \frac{\partial \Phi}{\partial \xi^{\prime}}
\end{array}\right| .
$$

If the magnitude of the deflected electric field is $S_{\text {deflected }}(\Theta, \Phi)$ as in Eq. (3) and the Fresnel reflection and transmission coefficients are temporarily ignored, flux conservation for the incident and the deflected bundles of rays gives

$$
S_{\text {deflected }}(\Theta, \Phi)=\left(\frac{k^{2} A B r^{\prime}}{\Delta \sin \Theta}\right)^{1 / 2} .
$$

In Subsection 3.B., Eq. (18) is evaluated for specular reflection by the arbitrarily oriented spheroid.

\section{B. Magnitude of the Reflected Electric Field}

The geometry of a light ray specularly reflected by a spheroid is shown in Fig. 6 . The unit wave vector of the incident ray is

$$
\hat{k}_{\text {inc }}=\hat{u}_{z}{ }^{\prime},
$$

and the unit wave vector of the reflected ray referenced with respect to the rotated lab coordinates $x^{\prime} y^{\prime} z^{\prime}$ is ${ }^{37}$

$$
\begin{aligned}
\hat{k}_{\mathrm{ref}}= & \hat{k}_{\mathrm{inc}}-2 \hat{m}^{\prime}\left(\hat{k}_{\mathrm{inc}} \cdot \hat{m}^{\prime}\right)=\sin \Theta \cos (\Phi-\phi) \hat{u}_{x}{ }^{\prime} \\
& +\sin \Theta \sin (\Phi-\phi) \hat{u}_{y}{ }^{\prime}+\cos \Theta \hat{u}_{z}{ }^{\prime} .
\end{aligned}
$$

The calculation of the magnitude of the reflected

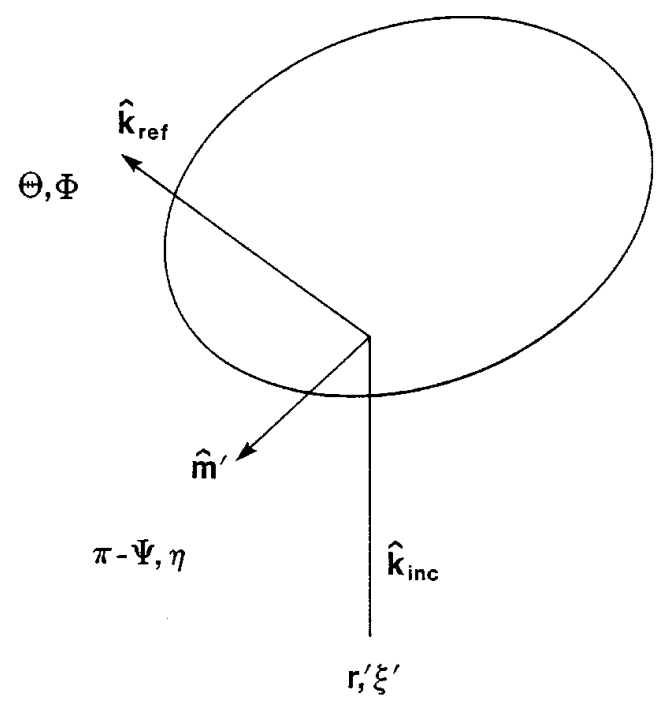

Fig. 6. Geometry of the reflected ray. The normal $\hat{m}^{\prime}$ to the surface at the point of incidence is in the $\pi-\Psi, \eta$ direction with respect to the $x^{\prime} y^{\prime} z^{\prime}$ rotated lab coordinate system. The incident and reflected unit wave vectors are $\hat{k}_{\text {inc }}$ and $\hat{k}_{\text {ref }}$, respectively. 
field $S_{\text {ref }}(\Theta, \Phi)$ proceeds in the following way. First we use Eq. (20) to obtain $\Theta$ and $\Phi$ for the outgoing ray in terms of $r^{\prime}$ and $\xi^{\prime}$ for the incoming ray. Next we invert these expressions to get $r^{\prime}$ and $\xi^{\prime}$ in terms of $\Theta$ and $\Phi$. We then perform the derivatives in Eq. (17) to obtain $S_{\text {ref }}$ in terms of $r^{\prime}$ and $\xi^{\prime}$. Last, we eliminate $r^{\prime}$ and $\xi^{\prime}$ from these expressions to obtain $S_{\text {ref }}$ in terms of $\Theta$ and $\Phi$, which is our desired goal.

As in Fig. 6, let the outward normal $\hat{m}^{\prime}$ to the lit spheroid surface be in the $\pi-\Psi, \eta$ direction referenced with respect to the $x^{\prime} y^{\prime} z^{\prime}$ rotated lab coordinate system and define $q^{\prime}$ as

$$
q^{\prime}=\frac{r^{\prime}}{\left(1-r^{\prime 2}\right)^{1 / 2}}
$$

or

$$
r^{\prime}=\frac{q^{\prime}}{\left(1+q^{\prime 2}\right)^{1 / 2}} .
$$

$$
q^{\prime}=\frac{A^{2}}{a b}\left[\left(\frac{B}{A} \tan \Psi \sin \eta\right)^{2}+(\tan \Psi \cos \eta-w)^{2}\right]^{1 / 2} .
$$

The angles $\Psi$ and $\eta$ of the normal to the spheroid surface are related to the scattering angles $\Theta$ and $\Phi$ of the reflected ray in the following way. Because $\hat{k}_{\text {inc }}, \hat{m}^{\prime}$, and $\hat{k}_{\text {ref }}$ lie in a single plane, we have

$$
\Phi-\phi=\eta \text {. }
$$

Then evaluating the $z^{\prime}$ component of Eq. (20), we obtain

$$
\cos \Theta=1-2\left(\hat{m} \cdot \hat{u}_{z}^{\prime}\right)^{2}
$$

or

$$
\Theta=\pi-2 \Psi .
$$

By combining Eqs. (22), (25)-(27), and (29), we obtain $r^{\prime}$ and $\xi^{\prime}$ in terms of $\Theta$ and $\Phi$ :

$$
\begin{aligned}
r^{\prime 2}= & \frac{\cos ^{2} \frac{\Theta}{2}\left[1+\left(\frac{B^{2}}{A^{2}}-1\right) \sin ^{2}(\Phi-\phi)\right]-w \sin \Theta \cos (\Phi-\phi)+w^{2} \sin ^{2} \frac{\Theta}{2}}{\left.1+\left(\frac{B^{2}}{A^{2}}-1\right)\left[\sin ^{2}(\Phi-\phi)+\sin ^{2} \frac{\Theta}{2} \cos ^{2}(\Phi-\phi)\right]+\left(\frac{B^{4}}{A^{4}} \frac{b^{2}}{a^{2}}-\frac{B^{2}}{A^{2}}+w^{2}\right)\right) \sin ^{2} \frac{\Theta}{2}-w \sin \Theta \cos (\Phi-\phi)}, \\
\tan \xi^{\prime}= & \frac{\frac{B}{A} \cos \frac{\Theta}{2} \sin (\Phi-\phi)}{\cos \frac{\Theta}{2} \cos (\Phi-\phi)-w \sin \frac{\Theta}{2}} .
\end{aligned}
$$

Then from Eq. (14) we have

$$
\begin{aligned}
\tan \eta & =\left(\frac{\partial z_{\text {lower }}}{\partial y^{\prime}}\right) /\left(\frac{\partial z_{\text {lower }}}{\partial x^{\prime}}\right) \\
& =\frac{\frac{a b}{A B} q^{\prime} \sin \xi^{\prime}}{\frac{a b}{A^{2}} q^{\prime} \cos \xi^{\prime}+w}, \\
\tan ^{2}(\pi-\Psi) & =\left(\frac{\partial z_{\text {lower }}}{\partial x^{\prime}}\right)^{2}+\left(\frac{\partial z_{\text {lower }}}{\partial y^{\prime}}\right)^{2} \\
& =\left(\frac{a b}{A^{2}} q^{\prime} \cos \xi^{\prime}+w\right)^{2}+\left(\frac{a b}{A B} q^{\prime} \sin \xi^{\prime}\right)^{2} .
\end{aligned}
$$

Equations (23) and (24) can be inverted to give

$$
\tan \xi^{\prime}=\frac{\frac{B}{A} \tan \Psi \sin \eta}{\tan \Psi \cos \eta-w},
$$

In other words, given the direction of a reflected ray, we know which incident ray it corresponds to.

On the other hand, by combining Eqs. (21), (23) and (24), (27), and (29), we obtain $\Theta$ and $\Phi$ in terms of $r^{\prime}$ and $\xi^{\prime}$ :

$$
\begin{aligned}
\Theta= & \pi-2 \arctan \left[\left(\frac{a b}{A^{2}} q^{\prime} \cos \xi^{\prime}+w\right)^{2}\right. \\
& \left.+\left(\frac{a b}{A B} q^{\prime} \sin \xi^{\prime}\right)^{2}\right]^{1 / 2}, \\
\Phi= & \phi+\arctan \left(\frac{\frac{a b}{A B} q^{\prime} \sin \xi^{\prime}}{\frac{a b}{A^{2}} q^{\prime} \cos \xi^{\prime}+w^{2}}\right) .
\end{aligned}
$$

We can now use Eq. (31) to perform the derivatives required in Eq. (17) to obtain

$$
S_{\mathrm{ref}}(\Theta, \Phi)=\frac{k A}{2}\left(\frac{A B}{a b}\right) \frac{\left(1-r^{\prime 2}\right)}{\sin ^{2} \frac{\Theta}{2}} .
$$


Substituting Eq. (30) for $r^{\prime}$ then gives

$$
S_{\mathrm{ref}}(\Theta, \Phi)=\frac{k b}{2}\left(\frac{B^{2}}{A^{2}}\right) / F(\Theta, \Phi),
$$

where

$$
\begin{aligned}
F(\Theta, \Phi)= & 1+\left(\frac{B^{2}}{A^{2}}-1\right) \\
& \times\left[\sin ^{2}(\Phi-\phi)+\sin ^{2} \frac{\Theta}{2} \cos ^{2}(\Phi-\phi)\right] \\
& +\left(\frac{B^{4}}{A^{4}} \frac{b^{2}}{a^{2}}-\frac{B^{2}}{A^{2}}+w^{2}\right) \\
& \times \sin ^{2} \frac{\Theta}{2}-w \sin \Theta \cos (\Phi-\phi) .
\end{aligned}
$$

After much algebra, Eqs. (33) and (34) reduce to than diffraction by an aperture,${ }^{40}$ we obtain

$$
\begin{aligned}
S_{\text {diff }}(\Theta, \Phi)= & (k A)^{2} \frac{B}{A} \\
\times & \frac{J_{1}\left\{k A \Theta\left[\cos ^{2}(\Phi-\phi)+\frac{B^{2}}{A^{2}} \sin ^{2}(\Phi-\phi)\right]^{1 / 2}\right\}}{k A \Theta\left[\cos ^{2}(\Phi-\phi)+\frac{B^{2}}{A^{2}} \sin ^{2}(\Phi-\phi)\right]^{1 / 2}} .
\end{aligned}
$$

In Eq. (38) we replaced the $\sin \Theta$ factor of Eq. (36) by $\Theta$ in order to obtain a closer agreement with the results of Lorenz-Mie theory. ${ }^{40}$

\section{Phase of the Reflected Electric Field}

The path of the reference ray is shown in Fig. 7 . It travels along the $z$ axis to the origin as if the spheroid were absent. It then turns toward the $\Theta$, $\Phi$ direction and travels in that direction to the far

$$
S_{\mathrm{ref}}(\Theta, \Phi)=\frac{k b / 2}{1+\left(\frac{b^{2}}{a^{2}}-1\right)\left[\cos \frac{\Theta}{2} \cos (\Phi-\phi) \sin \theta-\sin \frac{\Theta}{2} \cos \theta\right]^{2}} .
$$

Equation (35) is our final result for the magnitude of the reflected electric field.

\section{Magnitude of the Diffracted Electric Field}

The method for calculating the diffracted electric field for the elliptical aperture of Eq. (11) is given in Ref. 38. The Fraunhofer diffracted electric field produced by a plane wave that is incident upon an elliptical aperture is ${ }^{39}$

$$
\begin{aligned}
E_{\text {diff }}(\Theta, \Phi)= & \frac{-i k E_{0}}{2 \pi R} \exp (i k R-i \omega t) \int_{\text {ellipse }} \mathrm{d} x \mathrm{~d} y \\
& \times \exp [-i k(x \sin \Theta \cos \Phi+y \sin \Theta \sin \Phi)] .
\end{aligned}
$$

Changing to the rotated and scaled lab coordinate system

$$
\begin{aligned}
& x^{\prime \prime \prime}=x^{\prime}, \\
& y^{\prime \prime \prime}=\frac{A}{B} y^{\prime}
\end{aligned}
$$

converts Eq. (36) to a circular-aperture Fraunhofer diffraction integral. After multiplying Eq. (36) by a factor of -1 because of Babinet's principle because we are interested in diffraction by an obstacle rather zone. The path of the specularly reflected ray in the $\Theta, \Phi$ direction is also shown in Fig. 7. The pathlength difference between the reflected ray and the reference ray is calculated between the spheroid

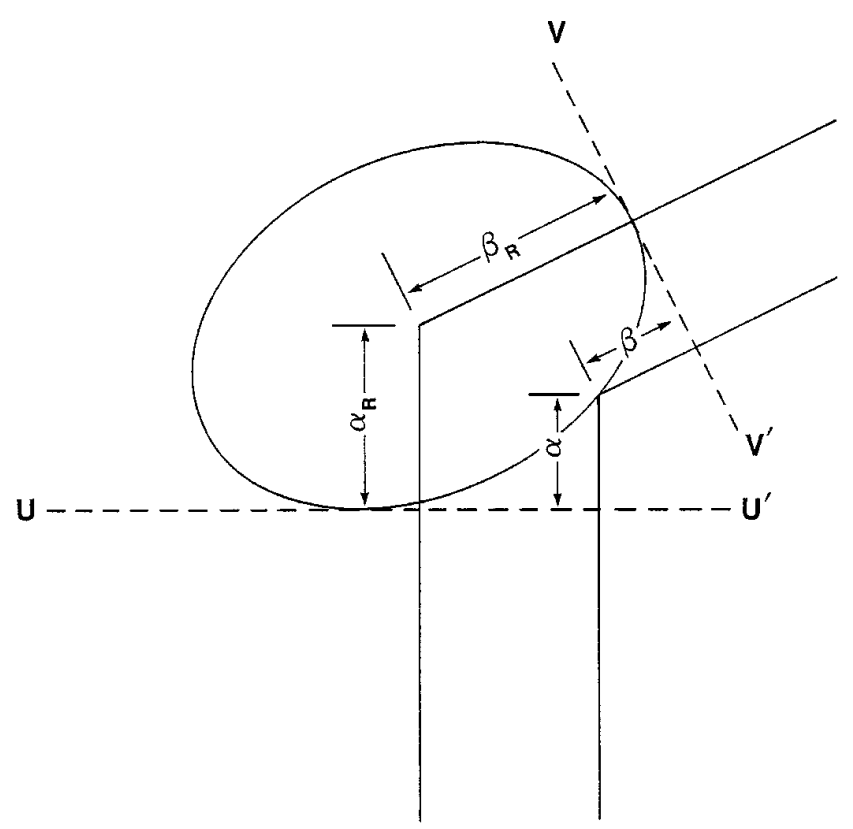

Fig. 7. Trajectories of the reflected ray and the reference ray. The spheroid entrance plane is $\mathrm{UU}^{\prime}$, and the spheroid exit plane is $\mathrm{VV}^{\prime}$. 
entrance and exit planes $\mathrm{UU}^{\prime}$ and $\mathrm{VV}^{\prime}$, respectively. The entrance plane is parallel to the $x y$ plane and touches the lowermost point of the spheroid $z_{\min }{ }^{\prime}<0$. The exit plane is normal to the outgoing reference ray and intersects it at the point where the reference ray crosses the spheroid surface. The exit plane is not necessarily tangent to the spheroid at this point. If the distances $\alpha$ and $\beta$ along the reflected ray and $\alpha_{R}$ and $\beta_{R}$ along the reference ray are defined as in Fig. 7 , the path-length difference between the reflected ray and the reference ray is $\alpha+\beta-\alpha_{R}-\beta_{R}$.

For the arbitrarily oriented spheroid and for an incident ray parameterized by $r^{\prime}, \xi^{\prime}$ we have

$$
\alpha=\alpha_{R}+z_{\text {lower }}{ }^{\prime}\left(r^{\prime}, \xi^{\prime}\right) .
$$

The distance $\beta_{R}$ is determined by finding the intersection of the outgoing reference ray,

$$
\begin{aligned}
& x^{\prime}=\beta_{R} \sin \Theta \cos (\Phi-\phi), \\
& y^{\prime}=\beta_{R} \sin \Theta \sin (\Phi-\phi), \\
& z^{\prime}=\beta_{R} \cos \Theta,
\end{aligned}
$$

for $\beta_{R} \geq 0$, with the upper or lower surface of the spheroid given by Eq. (13). The result is

$$
\begin{aligned}
\beta_{R}= & \left\{\frac{A^{2}}{a^{2} b^{2}}[\cos \Theta-w \sin \Theta \cos (\Phi-\phi)]^{2}+\sin ^{2} \Theta\right. \\
& \left.\times\left[\frac{\cos ^{2}(\Phi-\phi)}{A^{2}}+\frac{\sin ^{2}(\Phi-\phi)}{B^{2}}\right]\right\}^{-1 / 2}
\end{aligned}
$$

The equation of the plane normal to the line of Eq. (40) at the value of $\beta_{R}$ given by Eq. (41) is then obtained. The distance $\beta$ is obtained by finding the intersection of the reflected ray,

$$
\begin{aligned}
& x^{\prime}=A r^{\prime} \cos \xi^{\prime}+\beta \sin \Theta \cos (\Phi-\phi), \\
& y^{\prime}=B r^{\prime} \sin \xi^{\prime}+\beta \sin \Theta \sin (\Phi-\phi), \\
& z^{\prime}=z_{\text {lower }}{ }^{\prime}\left(r^{\prime}, \xi^{\prime}\right)+\beta \cos \Theta,
\end{aligned}
$$

for $\beta \geq 0$ with this plane. The result is

$$
\begin{aligned}
\beta= & \beta_{R}-A r^{\prime} \cos \xi^{\prime} \sin \Theta \cos (\Phi-\phi) \\
& -B r^{\prime} \sin \xi^{\prime} \sin \Theta \sin (\Phi-\phi) \\
& -z_{\text {lower }}\left(r^{\prime}, \xi^{\prime}\right) \cos \Theta .
\end{aligned}
$$

We claim that the phase of the reflected electric field of Eq. (3) is then

$$
\delta_{\text {ref }}(\Theta, \Phi)=\frac{2 \pi}{\lambda}\left(\alpha+\beta-\alpha_{R}-\beta_{R}\right)-\frac{\pi}{2},
$$

or, when Eqs. (39) and (43) are used and Eq. (30) is substituted for $r^{\prime}$ and $\xi^{\prime}$,

$$
\begin{aligned}
\delta_{\text {ref }}(\Theta, \Phi)= & \frac{2 \pi}{\lambda}\left[z_{\text {lower }}-A r^{\prime} \cos \xi^{\prime} \sin \Theta \cos (\Phi-\phi)\right. \\
& -B r^{\prime} \sin \xi^{\prime} \sin \Theta \sin (\Phi-\phi) \\
& \left.-z_{\text {lower }}{ }^{\prime} \cos \Theta\right]-\frac{\pi}{2} \\
= & \frac{-4 \pi a}{\lambda} \sin \frac{\Theta}{2}\left\{1+\left(\frac{b^{2}}{a^{2}}-1\right)\right. \\
& \times\left[\cos \frac{\Theta}{2} \cos (\Phi-\phi) \sin \theta\right. \\
& \left.\left.-\sin \frac{\Theta}{2} \cos \theta\right]^{2}\right\}^{1 / 2}-\frac{\pi}{2} .
\end{aligned}
$$

The first term of Eqs. (44) and (45) is the optical path-length difference between the reflected ray and the reference ray. The term ${ }^{41}-\pi / 2$ corresponds to $\exp (-i \pi / 2)=-i$ and serves to cancel the overall factor of $i$ in the definition of the scattered electric field in Eq. (3). This is necessary so that the reflected electric field at $\Theta=0^{\circ}$ for grazing incidence is real; i.e., for the transverse electric (TE) polarization the reflected electric field is out of phase by $180^{\circ}$ with the light rays that miss striking the spheroid and propagate undeflected in the forward direction, and for the transverse magnetic (TM) polarization the field is in phase with the rays that miss striking the spheroid.

There is an additional shift of $\pi / 2$ in the phase of a geometric light ray each time it participates in a focusing caustic either inside the dielectric particle or in the near zone or far zone after being deflected by it. ${ }^{41}$ For scattering by a sphere the usual prescription for the number of caustic participations given by van de Hulst is equivalent to the following. For the ray that is incident along the sphere axis, there is a focusing contribution to $\delta$ of 0 for reflection, $-\pi$ for transmission, and $(-\mathrm{p}+1) \pi$ for $p \geq 2$. For incident rays progressively farther from the sphere axis, this extra phase factor shifts by $-\pi / 2$ when the scattering angle passes through the rainbow angle, and it shifts by $+\pi / 2$ each time the scattering angle passes through either $0^{\circ}$ for a forward glory or $180^{\circ}$ for a backscattering glory. For reflection by a spheroid, there is no interior caustic because the reflected ray never enters the spheroid. There is no far-zone caustic because Eq. (32) never diverges. There is also no near-zone reflection caustic because of the convex shape of the lower spheroid surface. ${ }^{42}$ Thus for reflection there is no additional contribution to the phase beyond the result given in Eq. (45).

\section{E. Phase of the Diffracted Electric Field}

By comparing Eq. (3) with Eq. (36) amended by the extra factor of -1 because of Babinet's principle, we 
find that

$$
\delta_{\text {diff }}=0 .
$$

\section{F. Polarization of the Reflected Electric Field}

The incident electric field of Eq. (1) may be written as

$$
\mathbf{E}_{\text {inc }}=E_{0} \exp (i k z-i \omega t) \hat{\epsilon}_{\text {inc }},
$$

where the incident-beam polarization vector is

$$
\begin{aligned}
\hat{\epsilon}_{\text {inc }} & =\cos \chi \hat{u}_{x}+\sin \chi \hat{u}_{y} \\
& =\cos (\chi-\phi) \hat{u}_{x}{ }^{\prime}+\sin (\chi-\phi) \hat{u}_{y}{ }^{\prime} .
\end{aligned}
$$

At the point where the incident ray strikes the lower spheroid surface, the polarization vector $\hat{\epsilon}_{\text {inc }}$ may be decomposed into components in the TE and TM directions. For the TE polarization (also called the $s$ polarization), the electric field of the incident ray is parallel to the plane tangent to the spheroid at the point of incidence. For the TM polarization (also called the $p$ polarization), the magnetic field of the incident ray is parallel to the tangent plane. The unit vectors in the TE and TM directions are then

$$
\begin{aligned}
& \widehat{\mathrm{TE}}_{\mathrm{inc}}=\frac{\hat{m}^{\prime} \times \hat{k}_{\mathrm{inc}}}{\sin \theta_{\mathrm{inc}}}=\sin \eta \hat{u}_{x}{ }^{\prime}-\cos \eta \hat{u}_{y}{ }^{\prime}, \\
& \widehat{\mathrm{TM}}_{\mathrm{inc}}=\frac{\hat{k}_{\mathrm{inc}} \times\left(\hat{m}^{\prime} \times \hat{k}_{\mathrm{inc}}\right)}{\sin \theta_{\mathrm{inc}}}=\cos \eta \hat{u}_{x}{ }^{\prime}+\sin \eta \hat{u}_{y}{ }^{\prime},
\end{aligned}
$$

respectively. The angle of incidence of the light ray at the spheroid surface is $\theta_{\text {inc }}$, and its cosine is given by

$$
\hat{k}_{\text {inc }} \cdot \hat{m}^{\prime}=\cos \theta_{\text {inc }}=\cos \Psi .
$$

In terms of these new unit vectors, the polarization vector of the incident ray is

$$
\hat{\epsilon}_{\mathrm{inc}}=\cos \gamma \widehat{\mathrm{TE}}_{\mathrm{inc}}+\sin \gamma \widehat{\mathrm{TM}}_{\mathrm{inc}},
$$

where

$$
\gamma=\chi-\Phi-\frac{3 \pi}{2}
$$

After specular reflection, the unit vectors in the TE and TM directions for the outgoing reflected ray are

$$
\begin{aligned}
\widehat{\mathrm{TE}}_{\mathrm{ref}}= & \frac{\hat{m}^{\prime} \times \hat{k}_{\mathrm{ref}}}{\sin \theta_{\mathrm{ref}}}=\sin \eta \hat{u}_{x}{ }^{\prime}-\cos \eta \hat{u}_{y}{ }^{\prime}, \\
\widehat{\mathrm{TM}}_{\mathrm{ref}}= & \frac{\hat{k}_{\mathrm{ref}} \times\left(\hat{m}^{\prime} \times \hat{k}_{\mathrm{ref}}\right)}{\sin \theta_{\mathrm{ref}}}=\cos \Theta \cos \eta \hat{u}_{x}{ }^{\prime} \\
& +\cos \Theta \sin \eta \hat{u}_{y}{ }^{\prime}-\sin \Theta \hat{u}_{z}{ }^{\prime},
\end{aligned}
$$

respectively, where $\theta_{\text {ref }}$ is given by the specularreflection law

$$
\theta_{\text {inc }}=\theta_{\text {ref }}
$$

The polarization vector of the outgoing reflected ray is then

$$
\hat{\epsilon}_{\mathrm{ref}}=(\cos \gamma) r_{\mathrm{TE}} \widehat{\mathrm{TE}}_{\mathrm{ref}}+(\sin \gamma) r_{\mathrm{TM}} \widehat{\mathrm{TM}}_{\mathrm{ref}},
$$

where $r_{\mathrm{TE}}\left(\theta_{\text {inc }}\right)$ and $r_{\mathrm{TM}}\left(\theta_{\text {inc }}\right)$ are the $\mathrm{TE}$ and $\mathrm{TM}$ Fresnel coefficients for reflection, respectively. ${ }^{43}$

\section{G. Polarization of the Diffracted Electric Field}

In this section the polarization of the electric field diffracted by a sphere is calculated with the Debye series decomposition of the partial-wave scattering amplitudes in Lorenz-Mie theory. ${ }^{44}$ It is then conjectured that the diffracted-field polarization for spheroid scattering is constructed in the same way. Diffraction by a spherical obstacle is produced by an interaction of the grazing incident rays with the sphere surface..$^{40}$ Consider a grazing incident ray parameterized by the coordinates $r_{d}{ }^{\prime}=1$ and $\xi_{d}{ }^{\prime}$, where $0 \leq \xi_{d}{ }^{\prime}<2 \pi$. In Subsections 3.B., 3.D., and 3.F., the absence of subscripts for $r^{\prime}$ and $\xi^{\prime}$ means that they describe an incident ray that will be reflected into the $\Theta, \Phi$ direction. The presence of the subscript $d$ in this section denotes the coordinates $r^{\prime}$ and $\xi^{\prime}$ for an incident ray that will be diffracted into the $\Theta, \Phi$ direction. The normal to the sphere surface at a grazing incidence point is

$$
\hat{m}_{d}{ }^{\prime \text { sphere }}=\cos \xi_{d}{ }^{\prime} \hat{u}_{x}{ }^{\prime}+\sin \xi_{d}{ }^{\prime} \hat{u}_{y}{ }^{\prime} .
$$

The incident TE and TM unit vectors for diffraction are then

$$
\widehat{\mathrm{TE}}_{i d}{ }^{\text {sphere }}=\hat{m}_{d}{ }^{\text {sphere }} \times \hat{k}_{\mathrm{inc}}=\sin \xi_{d}{ }^{\prime} \hat{u}_{x}{ }^{\prime}-\cos \xi_{d}{ }^{\prime} \hat{u}_{y}{ }^{\prime},
$$

$$
\begin{aligned}
\widehat{\mathrm{TM}}_{i d}{ }^{\text {sphere }} & =\hat{k}_{\text {inc }} \times\left(\hat{m}_{d}{ }^{\text {sphere }} \times \hat{k}_{\text {inc }}\right) \\
& =\cos \xi_{d}{ }^{\prime} \hat{u}_{x}{ }^{\prime}+\sin \xi_{d}{ }^{\prime} \hat{u}_{y}{ }^{\prime} .
\end{aligned}
$$

(For a sphere we take $\phi=0$ so that the lab $x$ and $x^{\prime}$ axes coincide.) The polarization vector of an incident ray is then

$$
\begin{aligned}
\hat{\epsilon}_{\text {inc }}^{\text {sphere }} & =\cos \chi \hat{u}_{x}{ }^{\prime}+\sin \chi \hat{u}_{y}{ }^{\prime} \\
& =\cos \gamma_{d}{ }^{\text {sphere }} \widehat{T E}_{i d}^{\text {sphere }}+\sin \gamma_{d}{ }^{\text {sphere }} \widehat{T M}_{i d}{ }^{\text {sphere }},
\end{aligned}
$$

where

$$
\gamma_{d}{ }^{\text {sphere }}=\chi-\xi_{d}{ }^{\prime}-\frac{3 \pi}{2} .
$$

In the Debye series expansion of the Lorenz-Mie partial-wave scattering amplitudes $a_{l}$ and $b_{l}$, the portion of the amplitudes associated with diffraction is $^{44}$

$$
a_{l}^{\text {diff }}=b_{l}^{\text {diff }}=1 / 2
$$


Substituting this into the Lorenz-Mie expressions for the scattered electric field in the near-forward direction, where the angular functions are approximated by ${ }^{45}$

$$
\pi_{l}(\Theta) \approx \tau_{l}(\Theta)=\frac{l(l+1)}{2} J_{0}\left[\left(l+\frac{1}{2}\right) \Theta\right],
$$

and converting the sum over partial waves into the integral over an associated impact parameter, ${ }^{46}$ we obtain $^{40}$

$$
\begin{aligned}
\mathbf{E}_{\text {diff }}^{\text {sphere }}(\Theta, \Phi)= & \frac{i E_{0}}{k R} \exp (i k R-i \omega t) S_{\text {diff }}^{\text {sphere }} \\
& \times(\Theta, \Phi) \hat{\epsilon}_{\text {diff }}^{\text {sphere }}
\end{aligned}
$$

where

$$
S_{\text {diff }}^{\text {sphere }}(\Theta, \Phi)=(k a)^{2} \frac{J_{1}(k a \Theta)}{k a \Theta},
$$

in analogy with Eq. (38), and where

$$
\hat{\epsilon}_{\text {diff }}^{\text {sphere }}=\cos (\Phi-\chi) \hat{u}_{\Theta}-\sin (\Phi-\chi) \hat{u}_{\Phi} .
$$

If $\hat{k}_{\text {diff }}$ is the unit wave vector in the direction of the outgoing diffracted ray, the outgoing diffracted TE and TM unit vectors are

$$
\begin{aligned}
\widehat{\mathrm{TE}}_{f d}{ }^{\text {sphere }} & =\frac{\hat{m}_{d}{ }^{\text {sphere }} \times \hat{k}_{\text {diff }}}{\sin \Theta}=-\hat{u}_{\Phi}, \\
\widehat{\mathrm{TM}}_{f d}{ }^{\text {sphere }} & =\frac{\hat{k}_{\text {diff }} \times\left(\hat{m}_{d}{ }^{\text {sphere }} \times \hat{k}_{\text {diff }}\right)}{\sin \Theta}=\hat{u}_{\Theta},
\end{aligned}
$$

respectively, giving

$\hat{\epsilon}_{\text {diff }}^{\text {sphere }}=\cos \gamma_{d}^{\text {sphere }} \widehat{\mathrm{TE}}_{f d}^{\text {sphere }}+\sin \gamma_{d}^{\text {sphere }} \widehat{\mathrm{TM}}_{f d}^{\text {sphere }}$.

For scattering by a sphere, Eq. (70) shows that the diffracted-field polarization vector $\hat{\epsilon}_{d}$ sphere is identical to the incident-field polarization vector $\hat{\epsilon}_{\text {inc }}$, except that the incident TE and TM unit vectors have been replaced by the final TE and TM unit vectors. We may conjecture that for spheroid scattering the diffracted-field polarization vector is constructed in the same way. The details of the construction are as follows.

For scattering by a spheroid, the unit normal to the edge of the spheroid at $r_{d}{ }^{\prime}=1$ is

where

$$
\hat{m}_{d}{ }^{\prime}=\cos \eta_{d} \hat{u}_{x}{ }^{\prime}+\sin \eta_{d} \hat{u}_{y}{ }^{\prime},
$$

$$
\tan \eta_{d}=\frac{A}{B} \tan \xi_{d}{ }^{\prime} .
$$

The incident TE and TM unit vectors for diffraction are then given by

$$
\begin{aligned}
& \widehat{\mathrm{TE}}_{i d}=\hat{m}_{d}{ }^{\prime} \times \hat{k}_{\mathrm{inc}}=\sin \eta_{d} \hat{u}_{x}{ }^{\prime}-\cos \eta_{d} \hat{u}_{y}{ }^{\prime}, \\
& \widehat{\mathrm{TM}}_{i d}=\hat{k}_{\mathrm{inc}} \times\left(\hat{m}_{d}{ }^{\prime} \times \hat{k}_{\mathrm{inc}}\right)=\cos \eta_{d} \hat{u}_{x}{ }^{\prime}+\sin \eta_{d} \hat{u}_{y}{ }^{\prime},
\end{aligned}
$$

respectively. The polarization vector of the incident electric field is

$$
\hat{\epsilon}_{\mathrm{inc}}=\cos \gamma_{d} \widehat{\mathrm{TE}}_{i d}+\sin \gamma_{d} \widehat{\mathrm{TM}}_{i d}
$$

where

$$
\gamma_{d}=\chi-\phi-\eta_{d}-\frac{3 \pi}{2}
$$

Light rays diffracted by a straight edge fan out in the plane normal to that edge. ${ }^{47}$ Also, in the shortwavelength limit the edge of the spheroid appears locally straight. Because the normal to the point $\left(r_{d}{ }^{\prime}=1, \xi_{d}{ }^{\prime}\right)$ on the spheroid surface makes an angle $\eta_{d}$ with the $x^{\prime}$ axis of the rotated lab coordinate system, the scattering angle of $\Phi$ the diffracted ray is given by

$$
\Phi-\phi=\eta_{d}
$$

The TE and TM unit vectors for the outgoing diffracted ray are then

$$
\begin{aligned}
\widehat{\mathrm{TE}}_{f d}= & \frac{\hat{m}_{d}{ }^{\prime} \times \hat{k}_{\text {diff }}}{\sin \Theta}=\sin \eta_{d} \hat{u}_{x}{ }^{\prime}-\cos \eta_{d} \hat{u}_{y}{ }^{\prime}, \\
\widehat{\mathrm{TM}}_{f d}= & \frac{\hat{k}_{\text {diff }} \times\left(\hat{m}_{d}{ }^{\prime} \times \hat{k}_{\text {diff }}\right)}{\sin \Theta}=\cos \Theta \cos \eta_{d} \hat{u}_{x}{ }^{\prime} \\
& +\cos \Theta \sin \eta_{d} \hat{u}_{y}{ }^{\prime}-\sin \Theta \hat{u}_{z}{ }^{\prime},
\end{aligned}
$$

respectively, and the polarization vector of the diffracted ray becomes

$$
\hat{\epsilon}_{\text {diff }}=\cos \gamma_{d} \widehat{\mathrm{TE}}_{f d}+\sin \gamma_{d} \widehat{\mathrm{TM}}_{f d},
$$

where $\gamma_{d}$ is given by Eqs. (76) and (77) and the unit vectors are given by Eqs. (78) and (79).

\section{H. Diffracted Plus Reflected Electric Field}

A comparison of Eqs. (27) and (77) shows that the angle $\eta$ for the ray reflected in the $\Theta, \Phi$ direction is identical to the angle $\eta_{d}$ for the ray diffracted in the $\Theta, \Phi$ direction even though the coordinates $\left(r^{\prime}, \xi^{\prime}\right)$ and $\left(r_{d}{ }^{\prime}, \xi_{d}{ }^{\prime}\right)$ of the incident rays reflected and diffracted into the $\Theta, \Phi$ direction are different. As a result, the TE and TM unit vectors of Eqs. (54) and (55) and (78) and (79) are identical, and our final expression for the diffracted plus reflected electric field in ray 
theory is then

$$
\begin{aligned}
\mathbf{E}_{\mathrm{diff}+\mathrm{ref}}= & \frac{i E_{0}}{k R} \exp (i k R-i \omega t)\left\{\left[S_{\mathrm{ref}} \exp \left(i \delta_{\mathrm{ref}}\right) \cos \gamma r_{\mathrm{TE}}\right.\right. \\
& \left.+S_{\mathrm{diff}} \cos \gamma_{d}\right] \widehat{\mathrm{TE}}_{\mathrm{ref}}+\left[S_{\mathrm{ref}} \exp \left(i \delta_{\mathrm{ref}}\right) \sin \gamma r_{\mathrm{TM}}\right. \\
& \left.\left.+S_{\mathrm{diff}} \sin \gamma_{d}\right] \widehat{\mathrm{TM}}_{\mathrm{ref}}\right\} .
\end{aligned}
$$

In Eq. (81) $S_{\text {ref }}$ is given by Eq. (35), $S_{\text {diff }}$ is given by Eq. (38), $\delta_{\text {ref }}$ is given by Eq. (45), $\gamma$ is given by Eq. (53), $\gamma_{d}$ is given by Eqs. (76) and (77), and the unit vectors are given by Eqs. (54) and (55).

All the quantities in Eq. (81) are directly expressible in terms of the scattering angles $\Theta$ and $\Phi$. If the spheroid is impenetrable, Eq. (81) represents the entire scattered field in the ray-theory approximation. If the spheroid is transparent, however, the transmitted field must be added to this result in order to describe scattering in the short-wavelength limit in the near-forward direction accurately. For scattering by an impenetrable sphere it is known that the diffraction-reflection interference structure in the Lorenz-Mie-scattered intensity damps out much faster as a function of scattering angle than the ray-theory intensity does. ${ }^{48}$ This is shown in Fig. 8 and indicates that for large scattering angles the actual diffracted electric field dies off faster than the paraxial approximation of Eq. (66). This more rapid die off is correctly explained with the complex angular momentum method. ${ }^{48-51}$ Although the complex angular-momentum method produces a marked improvement over the results of ray theory for scattering by a sphere in the short-wavelength limit, it is our point of view that ray theory provides a sensible zeroth-order approximation to short-wavelength scattering. This has been shown to be the case for scattering by a sphere, ${ }^{26,27}$ and we believe it to be the case for scattering by a spheroid as well. Ray theory thus complements the exact methods for spheroid scattering described in Section 1, because ray theory is expected to be reasonable beginning at approximately the spheroid-size parameter at which the numerical implementation of the exact methods starts becoming ill conditioned.

\section{Discussion}

The magnitude of the reflected electric field of Eq. (35) has a peculiar property. For backscattering with $\Theta=180^{\circ}$ we have

$$
S_{\mathrm{ref}}\left(\Theta=180^{\circ}, \Phi\right)=\frac{k b / 2}{1+\left(\frac{b^{2}}{a^{2}}-1\right) \cos ^{2} \theta},
$$

which is independent of $\Phi$ as it should be. But for

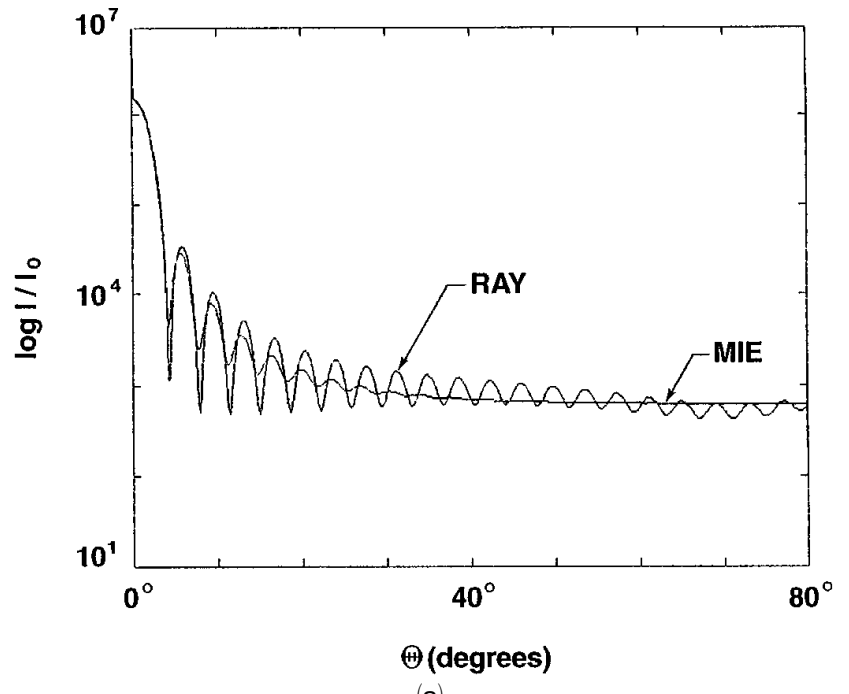

(a)

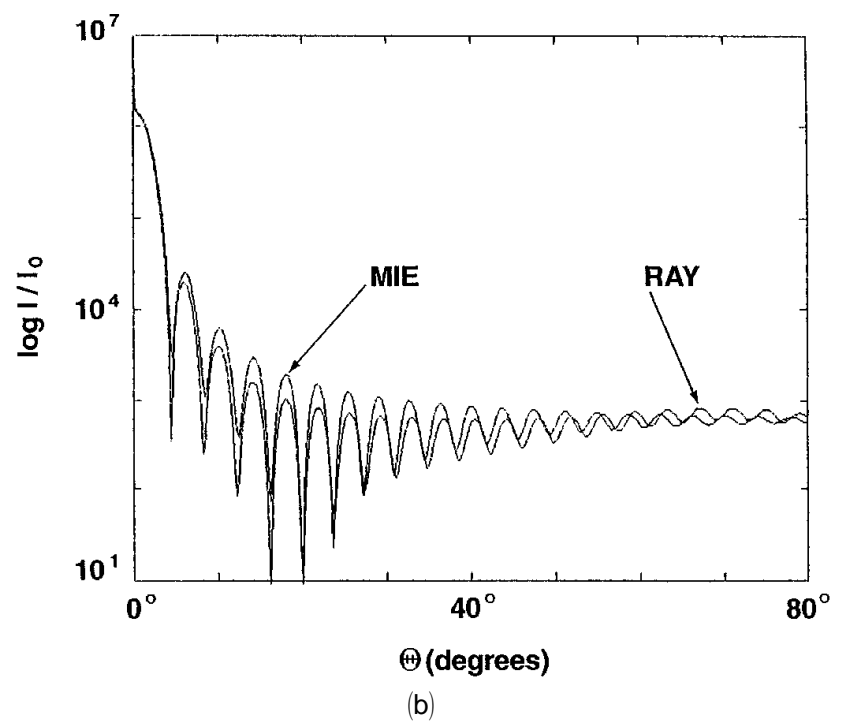

Fig. 8. Scattered intensity in Lorenz-Mie theory and ray theory for (a) the TE polarization and (b) the TM polarization as a function of the scattering angle $\theta$ for a conducting sphere with the size parameter $2 \pi a / \lambda=50$. The diffraction-reflection interference structure in the Lorenz-Mie intensity damps out much faster than in the ray-theory intensity. In this figure the incident intensity is given by $I_{0}=E_{0}^{2} / 2 \mu_{0} c$.

forward scattering with $\Theta=0^{\circ}$ we have

$$
S_{\mathrm{ref}}\left(\Theta=0^{\circ}, \Phi\right)=\frac{k b / 2}{1+\left(\frac{b^{2}}{a^{2}}-1\right) \cos ^{2}(\Phi-\phi) \sin ^{2} \theta},
$$

which is multivalued because $0 \leq \Phi<2 \pi$. This unconventional behavior is acceptable because the contribution of forward scattering to the far-zone reflected power,

$$
P_{\text {ref }}=\frac{E_{0}{ }^{2}}{2 \mu_{0} c} \int_{\Theta=0}^{\pi} \int_{\Phi=0}^{2 \pi} \frac{S_{\text {ref }}^{2}(\Theta, \Phi)}{k^{2}} \sin \Theta \mathrm{d} \Theta d \Phi,
$$


is zero because the solid angle at $\Theta=0^{\circ}$ is vanishingly small because of the sin $\Theta$ factor.

The multivalued nature of $S_{\mathrm{ref}}\left(\Theta=0^{\circ}, \Phi\right)$ can also be understood on physical grounds. Consider a point on the spheroid surface such that the normal to the surface at the point makes an angle $\Sigma$ with the spheroid major axis $z^{\prime \prime}$. The principal radii of curvature of the spheroid surface at the point are

$$
\begin{aligned}
& \rho_{1}=\frac{a^{2} b^{2}}{\left(a^{2} \sin ^{2} \Sigma+b^{2} \cos ^{2} \Sigma\right)^{3 / 2}}, \\
& \rho_{2}=\frac{a^{2}}{\left(a^{2} \sin ^{2} \Sigma+b^{2} \cos ^{2} \Sigma\right)^{1 / 2}},
\end{aligned}
$$

and the Gaussian curvature $K_{g}$ there is

$$
K_{g}=\left(\rho_{1} \rho_{2}\right)^{-1 / 2}=\frac{1+\left(\frac{b^{2}}{a^{2}}-1\right) \cos ^{2} \Sigma}{b} .
$$

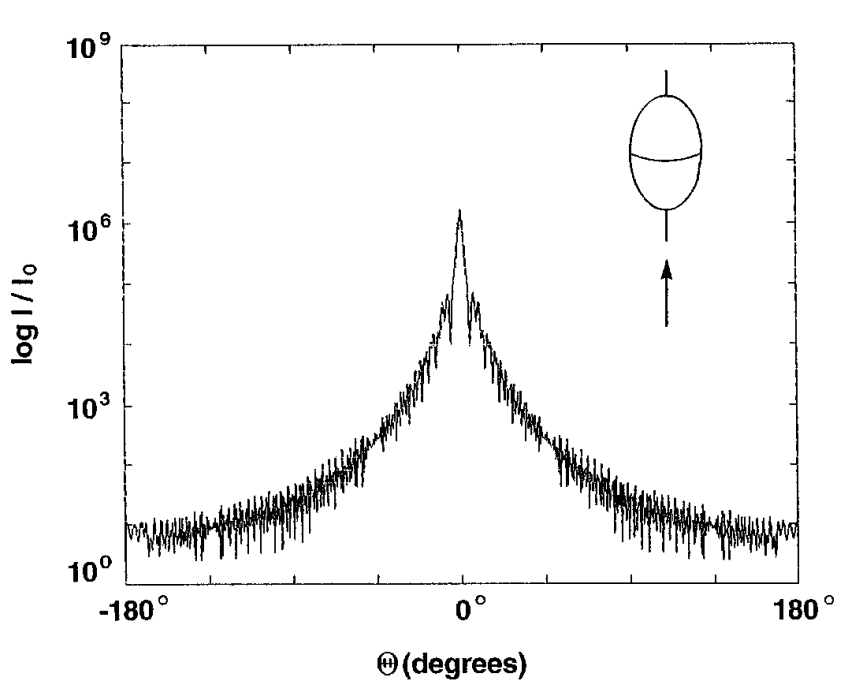

(a)

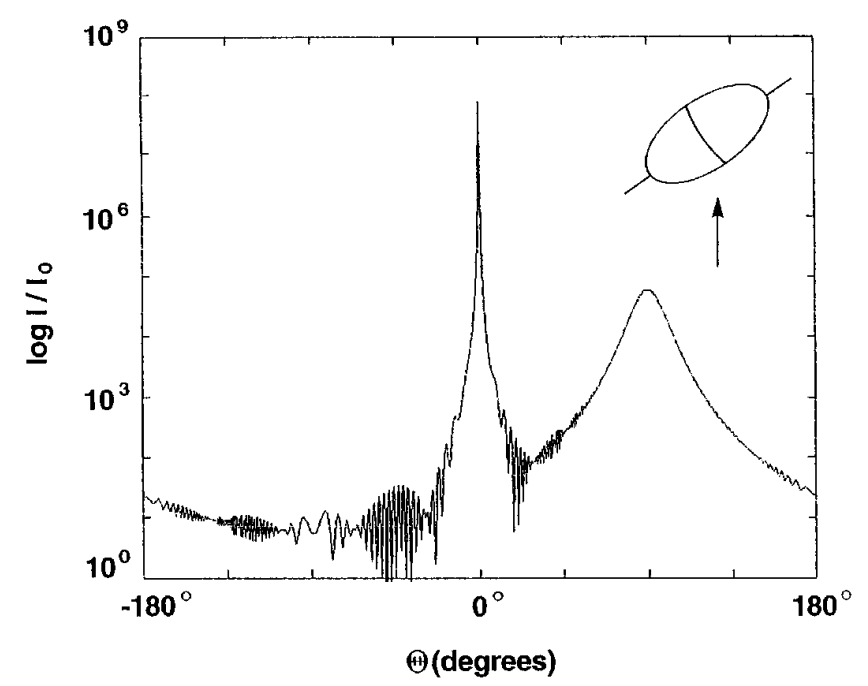

(b)
For the arbitrarily oriented spheroid, the unit vector in the $z^{\prime \prime}$ direction and the normal to the spheroid surface are related to the scattering angles $\Theta, \Phi$ by

$$
\begin{aligned}
\hat{u}_{z}{ }^{\prime \prime}= & \sin \theta \hat{u}_{x}{ }^{\prime}+\cos \theta \hat{u}_{z}{ }^{\prime}, \\
\hat{m}^{\prime}= & \cos \frac{\Theta}{2} \cos (\Phi-\phi) \hat{u}_{x}{ }^{\prime}+\cos \frac{\Theta}{2} \sin (\Phi-\phi) \\
& \times \hat{u}_{y}{ }^{\prime}-\sin \frac{\Theta}{2} \hat{u}_{z}{ }^{\prime},
\end{aligned}
$$

giving

$$
\begin{aligned}
\cos \Sigma & =\hat{u}_{z}{ }^{\prime \prime} \cdot \hat{m}^{\prime} \\
& =\cos \frac{\Theta}{2} \cos (\Phi-\phi) \sin \theta-\sin \frac{\Theta}{2} \cos \theta
\end{aligned}
$$

In general the magnitude of the reflected electric field is related to the Gaussian curvature of the
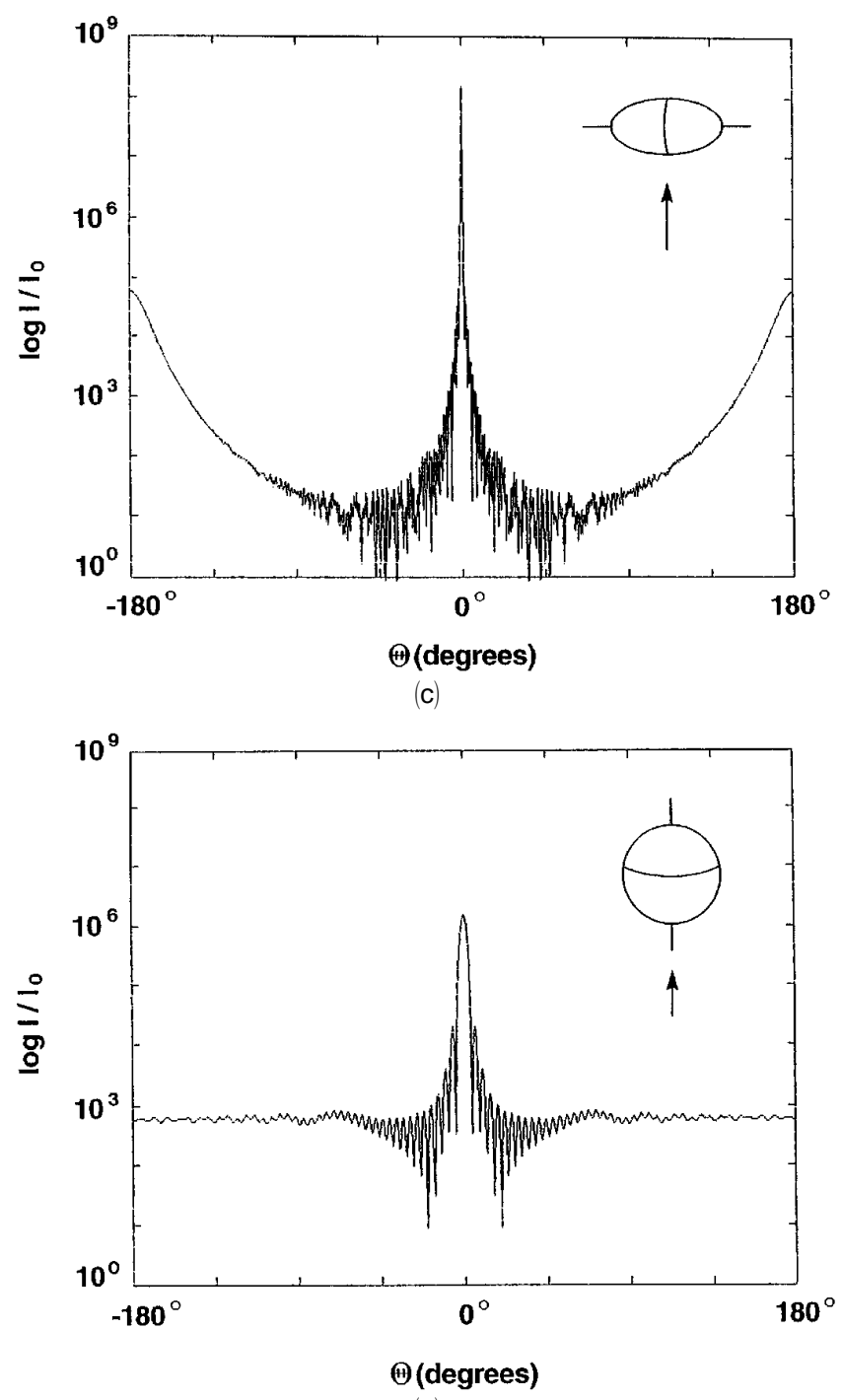

Fig. 9 (continued) 
reflecting surface for plane-wave incidence by ${ }^{42}$

$$
S_{\mathrm{ref}}=\frac{k}{2 K_{g}} .
$$

Substitution of Eqs. (87) and (89) into Eq. (90) immediately gives Eq. (35). A comparison with Eq. (45) also shows that $\delta_{\text {ref }}$ is simply related to $K_{g}$.

The light rays that are reflected at $\Theta=0^{\circ}$ graze the edge of the spheroid at the points on its circumference that are parameterized by $r^{\prime}=1$ and $0 \leq \xi^{\prime}<$ $2 \pi$. For reflection by a sphere or by a spheroid in the end-on orientation, all the points of grazing incidence have the same principal radii of curvature. As a result $S_{\text {ref }}\left(\Theta=0^{\circ}, \Phi\right)$ is independent of $\Phi$. But when the sphere is tilted with $\theta \neq 0^{\circ}$, the principal radii of curvature are different at different points of grazing incidence. This gives the $\Phi$ dependence to the magnitude of the forward-reflected electric field in Eq. (83).

As an example of ray theory applied to spheroid scattering, in Fig. 9 the ray-theory scattered inten-

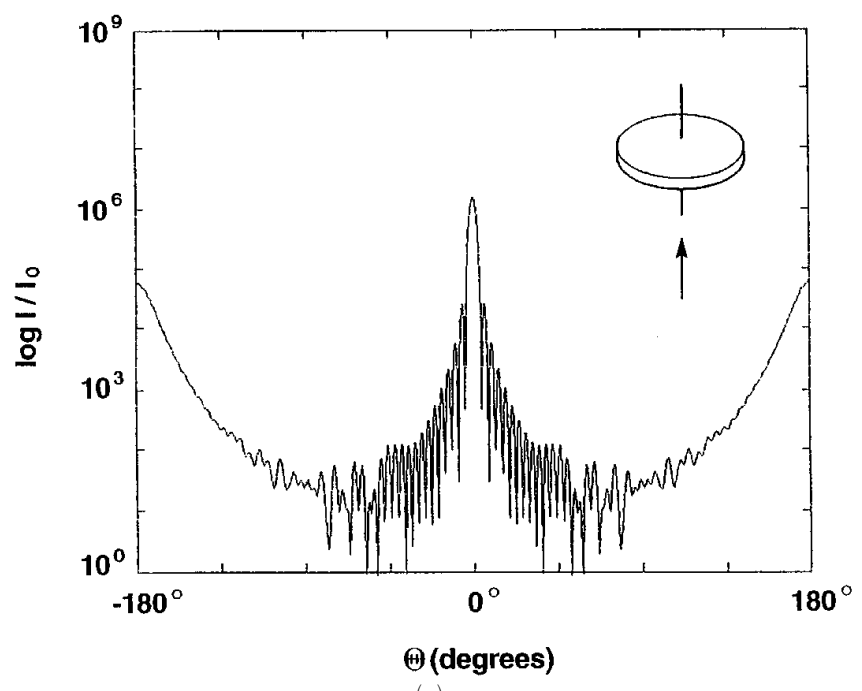

(e)

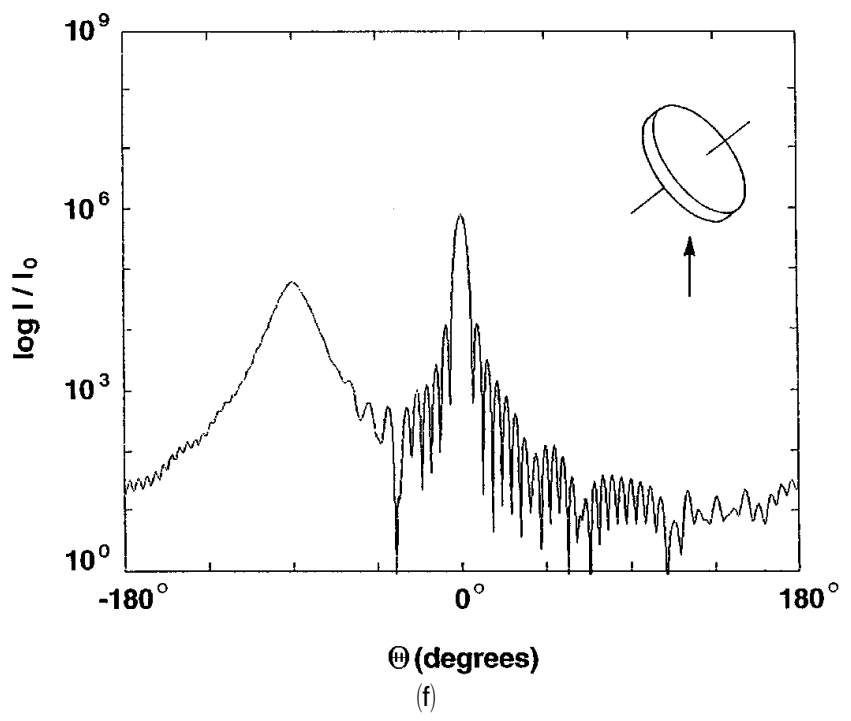

sity corresponding to Eq. (81) is graphed as a function of the scattering angle $\theta$ in the $\Phi=0^{\circ}$ plane for an incident plane wave with $\lambda=0.6328 \mu \mathrm{m}$ and $\chi=0^{\circ}$ striking a conducting spheroid with $a=5 \mu \mathrm{m}$ and variable $b / a$ whose major axis is tilted by a variable angle $\theta$ and rotated by $\phi=0^{\circ}$. Consider first Figs. $9(\mathrm{a}), 9(\mathrm{~d})$, and $9(\mathrm{e})$ for end-on incidence for a prolate spheroid, a sphere, and an oblate spheroid, respectively. In each of these cases the projection of the spheroid onto the $x y$ plane is a circle of radius $5 \mu \mathrm{m}$, giving an identical diffraction contribution. In Fig. $9(\mathrm{a})$, for $b / a=10$, the lower end of the prolate spheroid serves as a small target for the incident rays, leading to weak backscattering. Most of the rays glancingly strike the long sides of the spheroid, giving strong forward and near-forward reflection. In Fig. 9(d), for $b / a=1$, reflection by the sphere is isotropic, and the ripples in the intensity for $|\Theta| \geqslant$ $20^{\circ}$ are a result of the alternation between constructive and destructive interference of reflection and diffraction. In Fig. 9(e) for $b / a=0.1$, the lower flat platelike surface of the spheroid serves as a large

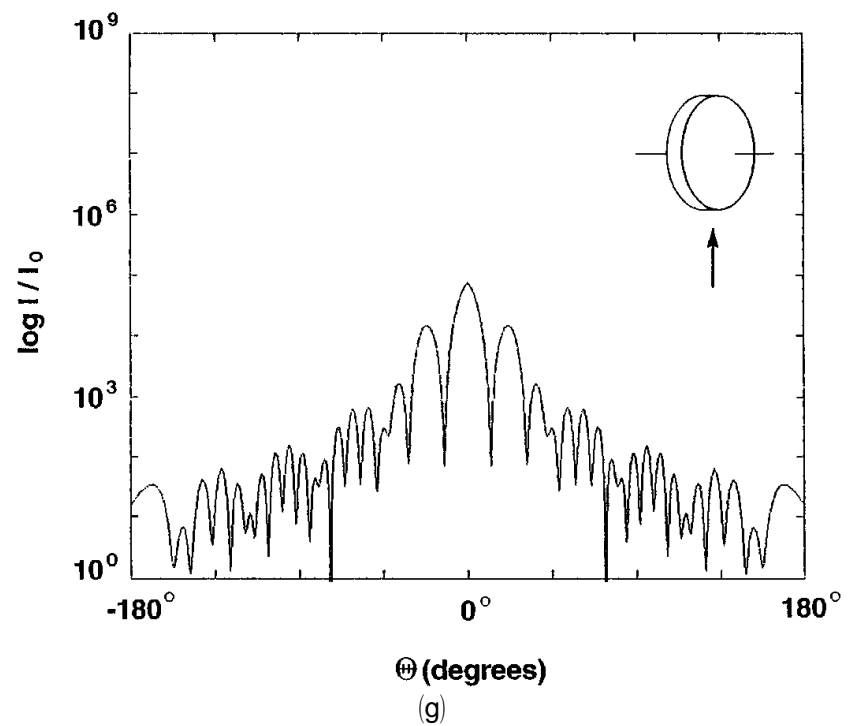

Fig. 9. Scattered intensity in ray theory for a plane wave with $\lambda=0.6328 \mu \mathrm{m}$ and $\chi=0^{\circ}$ incident upon a spheroid with $a=5 \mu \mathrm{m}$ as a function of the scattering angle $\Theta$ in the $\Phi=0^{\circ}$ plane. The spheroid parameters are (a) $b / a=10, \theta=0^{\circ}, \phi=0^{\circ}$; (b) $b / a=10$, $\theta=45^{\circ}, \phi=0^{\circ} ;$ (c) $b / a=10, \theta=90^{\circ}, \phi=0^{\circ}$ for a prolate spheroid being tilted from end-on incidence to side-on incidence; (d) $b / a=1$, $\theta=0^{\circ}, \phi=0^{\circ}$ for a sphere; and (e) $b / a=0.1, \theta=0^{\circ}, \phi=0^{\circ} ;(\mathrm{f})$ $b / a=0.1, \theta=45^{\circ}, \phi=0^{\circ} ;(\mathrm{g}) b / a=0.1, \theta=90^{\circ}, \phi=0^{\circ}$ for an oblate spheroid being tilted from end-on incidence to side-on incidence. In this figure the incident intensity is given by $I_{0}=$ $E_{0}{ }^{2} / 2 \mu_{0} c$. 
target for the incident rays, leading to strong backscattering. Relatively few rays strike the edge of the spheroid, leading to weak near-forward reflection.

Consider now Figs. 9(a)-9(c), which correspond to tilting the $b / a=10$ prolate spheroid from end-on incidence to side-on incidence. Because the projection of the tilted spheroid along the $x$ axis increases from $5 \mu \mathrm{m}$ in Fig. 9(a) to $50 \mu \mathrm{m}$ in Fig. 9(c), the diffraction peak narrows. More light rays reflect off of the long thin bottom of the spheroid than from anywhere else on its surface. This steers the strong reflected peak from $\Theta=0^{\circ}$ in Fig. 9 (a) to $\Theta=90^{\circ}$ in Fig. 9(b) and to $\Theta=180^{\circ}$ in Fig. 9(c).

Figures $9(\mathrm{e})-9(\mathrm{~g})$ correspond to tilting the $b / a=0.1$ oblate spheroid from end-on incidence to side-on incidence. Because the projection of the titled oblate spheroid along the $x$ axis decreases from $5 \mu \mathrm{m}$ in Fig. 9(e) to $0.5 \mu \mathrm{m}$ in Fig. $9(\mathrm{~g})$, the diffraction peak widens. The strong reflection peak is produced by rays striking the flat platelike lower surface of the spheroid. This steers the reflection peak from $\Theta=$ $180^{\circ}$ in Fig. $9(\mathrm{e})$ to $\Theta=-90^{\circ}$ in Fig. $9(\mathrm{f})$ and to $\Theta=0^{\circ}$ in Fig. $9(\mathrm{~g})$. For this geometry there is also a strong diffraction contribution centered on the reflection peak because the edge of the highly oblate spheroid serves as an effective aperture for the reflected rays. ${ }^{52}$ This additional diffraction effect has not been considered in this paper.

One can also examine scattering in the $\Phi=90^{\circ}$ plane by an prolate spheroid that has $a=5 \mu \mathrm{m}$ and $b / a=10$ and is tilted at $\theta=90^{\circ}, \phi=0^{\circ}$. Scattering in this plane resembles scattering at normal incidence by a conducting circular cylinder of finite length. In this orientation, the incident light rays see the circular cross section of the cylinder. As a result, the scattered intensity is identical to that of Fig. 9(d), except that everywhere it is a factor of $10^{2}$ larger. For $b / a \gg 1$ in general, Eq. (81) can be used to model scattering by a long conducting fiber. ${ }^{53,54}$ Similarly, one can examine scattering in the $\Phi=90^{\circ}$ plane by an oblate spheroid that has $a=5 \mu \mathrm{m}$ and $b / a=0.1$ and is tilted at $\theta=90^{\circ}, \phi=0^{\circ}$. The light rays that are incident along the $y$ axis reflect from the circular cross section of the tilted platelike spheroid. As a result, the scattered intensity is again identical to that of Fig. 9(d) except that is everywhere a factor of $10^{2}$ lower. For $b / a \ll 1$ in general, Eq. (81) can be used to model scattering by a flat conducting circular plate. ${ }^{52,55}$

Last, the formulas for the reflected and the diffracted electric fields for end-on incidence are examined, where $\theta=0^{\circ}, \phi=0^{\circ}$, so that $w=0$ in Eq. (8) and $A=B=a$ in Eqs. (9) and (10). The incident rays travel parallel to the major axis of the spheroid, and its projection in the $x y$ plane is a circle of radius $a$. For this case we have

$$
S_{\mathrm{ref}}(\Theta, \Phi)=\frac{k b / 2}{1+\left(\frac{b^{2}}{a^{2}}-1\right) \sin ^{2} \frac{\Theta}{2}},
$$

$$
\begin{aligned}
\delta_{\mathrm{ref}}(\Theta, \Phi)= & \frac{-4 \pi a}{\lambda} \sin \frac{\Theta}{2} \\
& \times\left[1+\left(\frac{b^{2}}{a^{2}}-1\right) \sin ^{2} \frac{\Theta}{2}\right]^{1 / 2}-\frac{\pi}{2}, \\
S_{\text {diff }}(\Theta, \Phi)= & (k a)^{2} \frac{J_{1}(k a \Theta)}{k a \Theta} .
\end{aligned}
$$

Both of these magnitudes and the reflected phase are independent of $\Phi$, illustrating that the scattered light has the same rotational symmetry as the spheroid in this orientation. In addition, Eq. (23) gives $\eta=\xi^{\prime}$ and Eq. (72) gives $\eta_{d}=\xi_{d}{ }^{\prime}$. This means that the plane that contains the $z$ axis and a given incident ray also contains the normals to the spheroid surface at the points of incidence for both reflection and diffraction. Thus for end-on incidence the trajectory of a given ray is confined to a single plane.

This completes our treatment of the diffraction plus reflection of an arbitrarily polarized plane wave by an arbitrarily oriented spheroid in ray theory. Transmission and the associated issue of crosspolarization effects are examined in the companion paper. $^{36}$

This work was supported in part by the National Aeronautics and Space Administration grant number NCC 3-204. I wish to thank P. L. Marston of Washington State University for making available to me the papers by Ravey and Mazerone. ${ }^{13-15}$

\section{References}

1. H. C. van de Hulst, Light Scattering by Small Particles (Dover, New York, 1981), Chap. 9.

2. M. Kerker, The Scattering of Light and Other Electromagnetic Radiation (Academic, New York, 1969), Chap. 3.

3. C. F. Bohren and D. R. Huffman, Absorption and Scattering of Light by Small Particles (Wiley, New York, 1983), Chap. 4.

4. W. J. Wiscombe, "Improved Mie scattering algorithms," Appl. Opt. 19, 1505-1509 (1980).

5. Ref. 3, appendix A and Sec. 4.8.

6. S. Asano and G. Yamamoto, "Light scattering by a spheroidal particle,"Appl. Opt. 14, 29-49 (1975); 15, 2028(E) (1976).

7. S. Asano, "Light scattering properties of spheroidal particles," Appl. Opt. 18, 712-723 (1979).

8. J. P. Barton, "Internal and near-surface electromagnetic fields for a spheroidal particle with arbitrary illumination," Appl. Opt. 34, 5542-5551 (1995).

9. P. Barber and C. Yeh, "Scattering of electromagnetic waves by arbitrarily shaped dielectric bodies," Appl. Opt. 14, 28642872 (1975).

10. P. W. Barber and S. C. Hill, Light Scattering by Particles: Computational Methods (World Scientific, Singapore, 1990), Chap. 3.

11. J. P. Barton and D. R. Alexander, "Electromagnetic fields for an irregularly shaped, near-spherical particle illuminated by a focused laser beam," J. Appl. Phys. 69, 7973-7986 (1991).

12. G. R. Fournier and B. T. N. Evans, "Approximation to extinction efficiency for randomly oriented spheroids," Appl. Opt. 30, 2042-2048 (1991).

13. J.-C. Ravey and P. Mazerone, "Light scattering in the physical optics approximation: application to large spheroids," J. Opt. (Paris) 13, 273-282 (1982). 
14. J. C. Ravey and P. Mazerone, "Light scattering by large spheroids in the physical optics approximation: numerical comparison with other approximate and exact results," J. Opt. (Paris) 14, 29-41 (1983).

15. J. C. Ravey, "The first extrema in the radiation pattern of the light scattered by micrometer-sized spheres and spheroids," J. Colloid Interface Sci. 105, 435-446 (1985).

16. T. W. Chen, "High energy light scattering in the generalized eikonal approximation," Appl. Opt. 28, 4096-4102 (1989).

17. T. W. Chen and W. S. Smith, "Large-angle light scattering at large size parameters," Appl. Opt. 30, 6558-6560 (1992).

18. T. W. Chen, "Simple formula for light scattering by a large spherical particle," Appl. Opt. 32, 7568-7571 (1993).

19. B. R. Levy and J. B. Keller, "Diffraction by a spheroid," Can. J. Phys. 38, 128-144 (1960).

20. M. V. Berry and C. Upstill, "Catastrophe optics: morphologies of caustics and their diffraction patterns," Prog. Opt. 18, 257-346 (1980).

21. W. J. Humphreys, Physics of the Air (Dover, New York, 1964), Chap. 3.

22. R. T. Wang and H. C. van de Hulst, "Mie computations and the Airy approximation," Appl. Opt. 30, 106-117 (1991).

23. P. L. Marston and E. H. Trinh, "Hyperbolic umbilic diffraction catastrophe and rainbow scattering from spheroidal drops," Nature 312, 529-531 (1984).

24. P. L. Marston, "Transverse cusp diffraction catastrophe: some pertinent wave fronts and a Pearcey approximation to the wave field," J. Acoust. Soc. Am. 81, 226-232 (1987).

25. C. E. Dean and P. L. Marston, "Opening rate of the cusp diffraction catastrophe in light scattered by oblate spheroidal drops," Appl. Opt. 30, 3443-3451 (1991).

26. W. J. Glantschnig and S.-H. Chen, "Light scattering from water droplets in the geometrical optics approximation," Appl. Opt. 20, 2499-2509 (1981).

27. A. Ungut, G. Gréhan, and G. Gouesbet, "Comparisons between geometrical optics and Lorenz-Mie theory," Appl. Opt. 20, 2911-2918 (1981).

28. E. A. Hovenac, "Calculation of far-field scattering from nonspherical particles using a geometrical optics approach," Appl. Opt. 30, 4739-4746 (1991).

29. J. A. Lock and T. A. McCollum, "Further thoughts on Newton's zero-order rainbow," Am. J. Phys. 62, 1082-1089 (1994).

30. J. F. Nye, "Rainbow scattering from spheroidal drops-an explanation of the hyperbolic umbilic foci," Nature 312, 531-532 (1984).

31. P. L. Marston, "Cusp diffraction catastrophe from spheroids: generalized rainbows and inverse scattering," Opt. Lett. 10, $588-590$ (1985).
32. J. F. Nye, "Rainbows from ellipsoidal water drops," Proc. R. Soc. London Ser. A 438, 397-417 (1992).

33. P. L. Marston and G. Kaduchak, "Generalized rainbows and unfolded glories of oblate drops: organization for multiple internal reflections and extension of cusps into Alexander's dark band," Appl. Opt. 33, 4702-4713 (1994).

34. K. Sassen, "Angular scattering and rainbow formation in pendant drops,” J. Opt. Soc. Am. 69, 1083-1089 (1979).

35. G. P. Können, "Appearance of supernumeraries of the second rainbow in rain showers," J. Opt. Soc. Am. A 4, 810-816 (1987).

36. J.A. Lock, "Ray scattering by an arbitrarily oriented spheroid: II. Transmission and cross-polarization effects," Appl. Opt. 35, 515-531 (1996).

37. J. B. Keller and H. B. Keller, "Determination of reflected and transmitted fields by geometrical optics," J. Opt. Soc. Am. 40, 48-52 (1950).

38. M. Born and E. Wolf, Principles of Optics, 6th corrected ed. (Pergamon, New York, 1980), pp. 398-399.

39. J. W. Goodman, Introduction to Fourier Optics (McGraw-Hill, New York, 1968), equation (4.13).

40. J. A. Lock and E. A. Hovenac, "Diffraction of a Gaussian beam by a spherical obstacle," Am. J. Phys. 61, 698-707 (1993).

41. Ref. 1, pp. 206-207.

42. P. L. Marston, "Geometrical and catastrophe optics methods in scattering," Phys. Acoust. 21, 1-234 (1992), Sec. 2.8.

43. E. Hecht, Optics, 2nd ed. (Addison-Wesley, Reading, Mass., 1987), Sect. 4.3.2.

44. J. A. Lock, "Cooperative effects among partial waves in Mie scattering,” J. Opt. Soc. Am. A 5, 2032-2044 (1988).

45. Ref. 1, Sec. 12.32 .

46. K. W. Ford and J. A. Wheeler, "Semiclassical description of scattering," Ann. Phys. (NY) 7, 259-286 (1959).

47. Ref. 43 , figure 10.9

48. H. M. Nussenzveig, "Uniform approximation in scattering by spheres,” J. Phys. A 21, 81-109 (1988).

49. H. M. Nussenzveig, "High-frequency scattering by an impenetrable sphere,"Ann. Phys. (NY) 34, 23-95 (1965).

50. H. M. Nussenzveig and W. J. Wiscombe, "Diffraction as tunneling," Phys. Rev. Lett. 59, 1667-1670 (1987).

51. H. M. Nussenzveig and W. J. Wiscombe, "Complex angular momentum approximation to hard-core scattering," Phys. Rev. A 43, 2093-2112 (1991).

52. Ref. 3, Sec. 8.4.7.

53. J. J. Bowman, T. B. A. Senior, and P. L. E. Uslenghi, Electromagnetic and Acoustic Scattering by Simple Shapes (Hemisphere, New York, 1987), Chap. 14.

54. Ref. 1, Sec. 15.22.

55. Ref. 1, Sec. 16.22. 